\title{
Unravelling the Molecular Basis of High Affinity Nanobodies against HIV p24: In Vitro Functional, Structural, and in Silico Insights
}

\author{
Eleanor R. Gray, ${ }^{\dagger} \perp \odot$ Jennifer C. Brookes, ${ }^{\dagger, \perp}$ Christophe Caillat, ${ }^{\ddagger}, \perp$ Valérian Turbé, ${ }^{\dagger}$
}

Benjamin L. J. Webb, ${ }^{\S}$ Luke A. Granger, ${ }^{\S, 1]}$ Benjamin S. Miller, ${ }^{\dagger}$ Laura E. McCoy, ${ }^{\S}$ Mohamed El Khattabi, ${ }^{\prime \prime}$ C. Theo Verrips, ${ }^{\|}$Robin A. Weiss, ${ }^{8}$ Dorothy M. Duffy, ${ }^{\dagger}$ Winfried Weissenhorn, ${ }^{\dagger}$ and Rachel A. McKendry* ${ }^{*}+\odot$

${ }^{\dagger}$ London Centre for Nanotechnology, Division of Medicine and Department of Physics and Astronomy, University College London, 17-19 Gordon Street, London, WC1H 0AH, United Kingdom

"Univ. Grenoble Alpes, CEA, CNRS, IBS, 71 Avenue des Martyrs, Grenoble, 38000, France

${ }^{\S}$ Division of Infection and Immunity, University College London, The Cruciform Building, Gower Street, London, WC1E 6BT, United Kingdom

"QVQ Holding B.V., Yalelaan 1, 3584CL, Utrecht, The Netherlands

Supporting Information

\begin{abstract}
Preventing the spread of infectious diseases remains an urgent priority worldwide, and this is driving the development of advanced nanotechnology to diagnose infections at the point of care. Herein, we report the creation of a library of novel nanobody capture ligands to detect p24, one of the earliest markers of HIV infection. We demonstrate that these nanobodies, one tenth the size of conventional antibodies, exhibit high sensitivity and broad specificity to global HIV1 subtypes. Biophysical characterization indicates strong $690 \mathrm{pM}$ binding constants and fast kinetic on-rates, 1 to 2 orders of magnitude better than monoclonal antibody comparators. A crystal structure of the lead nanobody and p24 was obtained and used alongside

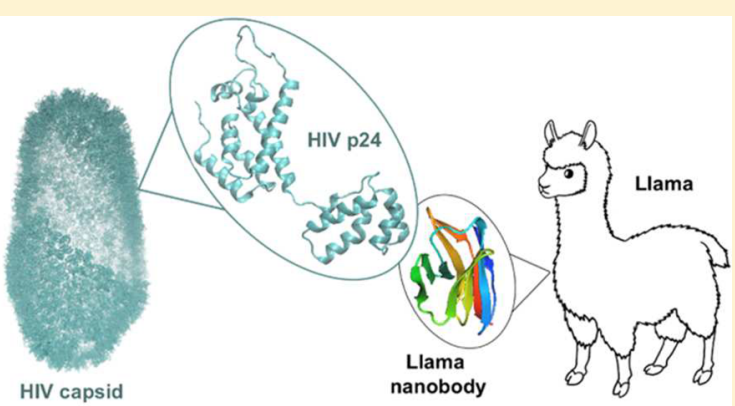
molecular dynamics simulations to elucidate the molecular basis of these enhanced performance characteristics. They indicate that binding occurs at $\mathrm{C}$-terminal helices 10 and 11 of p24, a negatively charged region of p24 complemented by the positive surface of the nanobody binding interface involving CDR1, CDR2, and CDR3 loops. Our findings have broad implications on the design of novel antibodies and a wide range of advanced biomedical applications.
\end{abstract}

KEYWORDS: HIV, nanobody, high-affinity, molecular dynamics, p24, crystal structure

$\mathrm{H}^{\mathrm{s}}$ IV is one of the fastest evolving of all organisms, presenting a challenge to the development of vaccines and diagnostics. Selection of a stable and consistent antigenic target is difficult due to the rapid rate of evolution, which is a combined effect of the high mutation rate and a recombination rate that is at the limit known for any organism. ${ }^{1}$ HIV-1 currently infects 34 million people worldwide, with high morbidity and mortality in Sub-Saharan Africa. ${ }^{2}$ However, even in the UK, more than one in five people are unaware of their infection, and $47 \%$ of diagnoses are at a late stage of AIDS. ${ }^{3}$ Early diagnosis plays a crucial role in all treatment and prevention strategies. It enables individual patients to gain faster access to care and treatment. ${ }^{4}$ In turn, this protects the wider public from the risk of subsequent infection, leading to major human and economic benefits to society. ${ }^{5,6}$ This has led to broad policy changes to widen access to care outside of centralized hospitals, including doctor's surgeries, pharmacies, community outreach settings, and the home, including selftesting, which is now legal in several countries including the US and $\mathrm{UK}^{7,8}$
The p24 capsid protein is the earliest protein biomarker of an HIV-1 infection and used in gold-standard HIV-1 fourth generation diagnostic tests. However, p24 detection is not widely used in point of care (POC) tests, which typically rely on just antibody detection, meaning that recent infections pass undetected before seroconversion, which can take 3-6 months. ${ }^{9}$ The first fourth generation POC test (detecting p24 and anti-HIV antibodies) to be approved has shown variable results for $\mathrm{p} 24$ detection in the field. ${ }^{10}$ The reasons behind this failure are unclear, but POC testing in resource-limited settings poses particular challenges, with little sophisticated laboratory instrumentation, no inbuilt immune complex disruption to dissociate p24 from complexing host antibodies, and no cold chain transportation to maintain reagent stability. ${ }^{11}$ This highlights the need for more sensitive, specific, and robust

Received: November 7, 2016

Published: June 7, 2017 


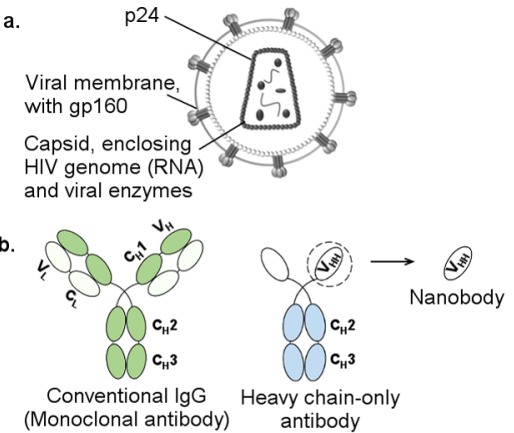

c.

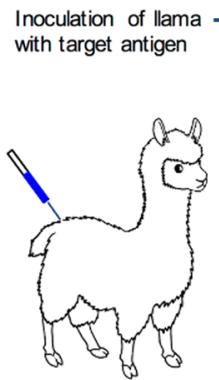

Isolation of B-lymphocytes and extraction of RNA

Amplification of $\mathrm{VHH}$ repertoire, Target p24 antigen immobilization Selection by phage display for binding to target antigen Second round of selection by phage display for binding to target antigen Random selection of clones, purification of individual nanobodies Positive selection by screening for binding to antigen Negative selection by screening for binding to distantly related antigens Characterization of selected nanobodies
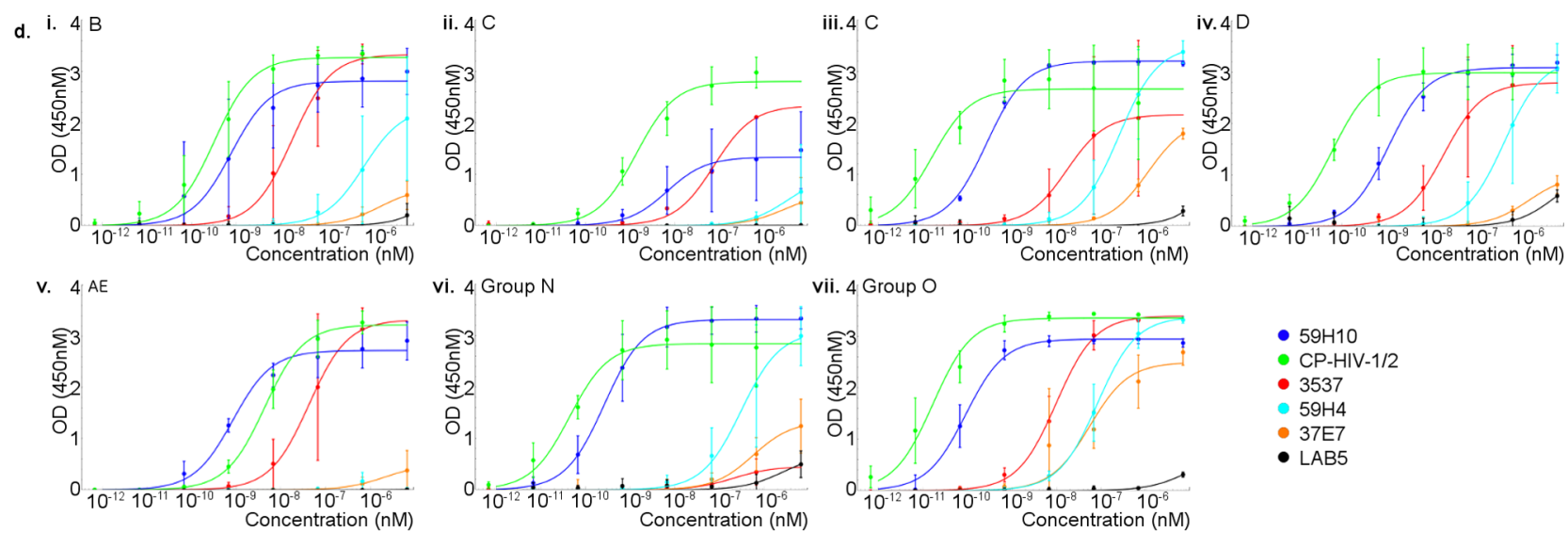

vii. 4 Group 0
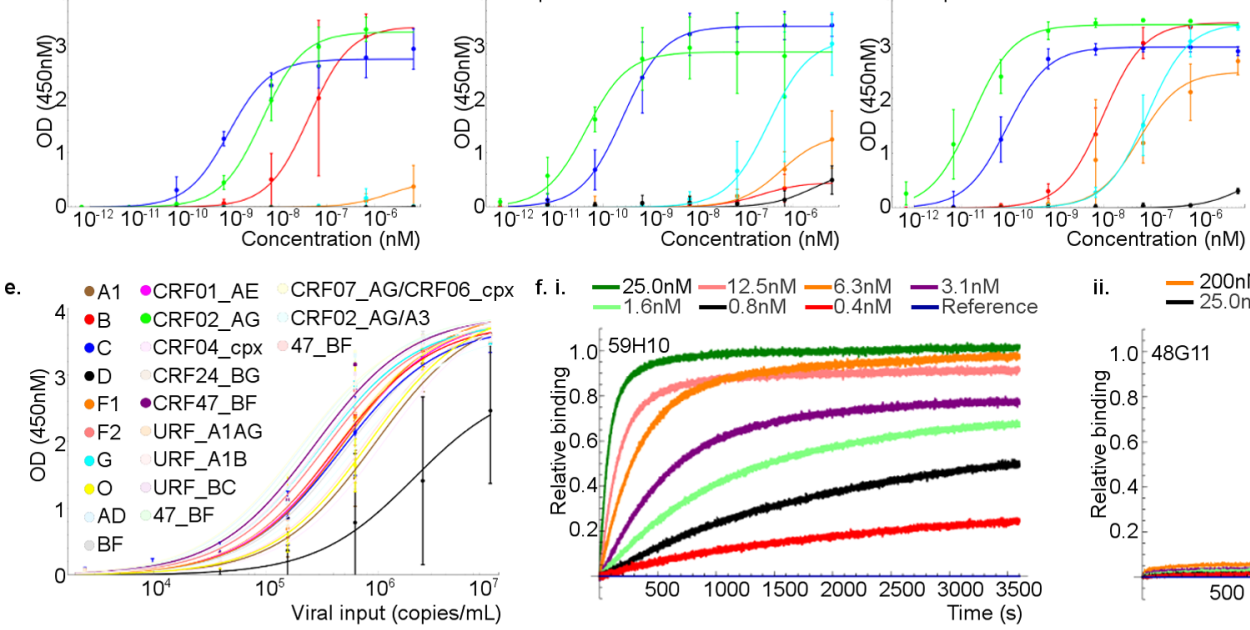

Concentration $(\mathrm{nM})$

iii. $\quad \underset{-800 \mathrm{nM}}{ }=400 \mathrm{nM}=200 \mathrm{nM}=100 \mathrm{nM}$

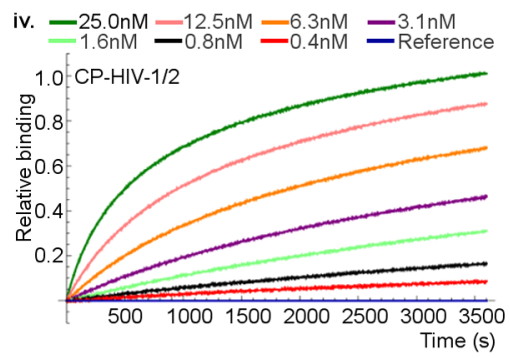

ii. $\quad=200 \mathrm{nM}=100 \mathrm{nM}=50.0 \mathrm{nM}$
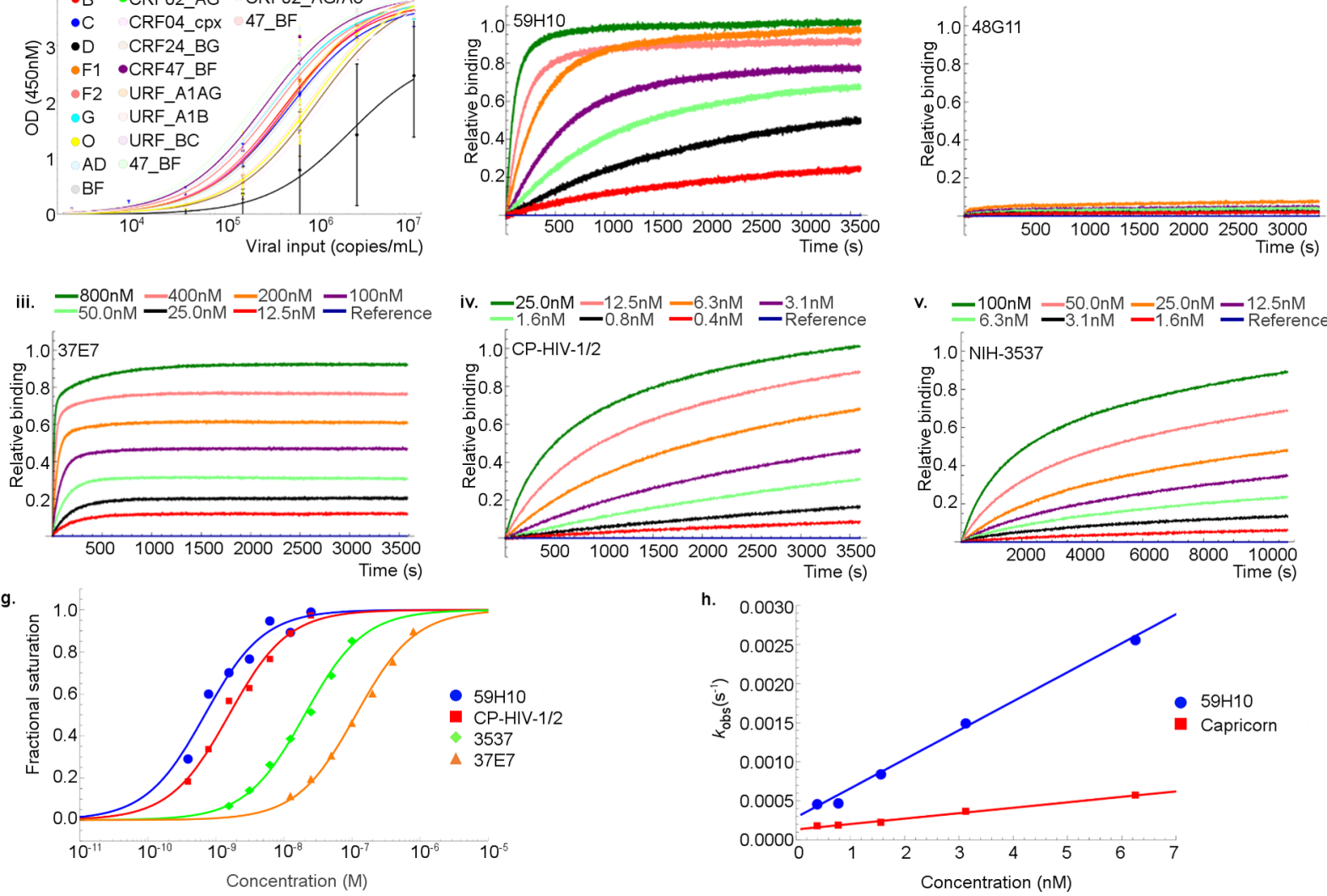

Figure 1. Schematics to show the structure of HIV, antibodies, and nanobodies, the method of generation of our p24 nanobodies, and characterization of thermodynamic and kinetic binding measurements for nanobodies and mAb comparators. (a) Schematic of a virion of HIV highlighting key antigens; (b) Schematic to compare the structures of a conventional mAb, heavy chain only antibody, and a nanobody with indicated domain substructure. $\mathrm{CH}$, constant heavy; $\mathrm{VH}$, variable heavy; $\mathrm{CL}$, constant light; VL, variable light; VHH, variable domain of a heavy chain-only antibody; (c) Outline of the phage library construction, screening, and nanobody selection after inoculation of a llama with target antigen, p24 (this process is described in more detail in ref 32); (d) Plots to show the comparison of binding of different p24 subtypes by four nanobodies and two p24-binding mAbs. Shown are the results from an indirect ELISA for subtype B (EVA620), subtype C (ARP695), subtype C (92BR025), 
Figure 1. continued

subtype D (NDK), and subtype AE (CM240), representative of Group N (06CM-U14296) and representative of Group O (BCF06) with nanobodies 59H10, 37E7, 59H4 and LAB5, and mAbs NIH-3537 and Capricorn HIV-1/2 (CP-HIV-1/2). LAB5 is a control nanobody that was raised to a phage protein. ${ }^{33}$ Shown is the molarity of antibody or nanobody versus the OD achieved (at $450 \mathrm{~nm}$ ). Results are combined from at least two experiments. (e) A sandwich ELISA was performed to assess detection of 50 viral strains from the EQAPOL viral subtype diversity panel. The ELISA was in a sandwich format with a mAb capture antibody (CP-HIV-1/2) and 59H10 detection nanobody. Error bars show standard deviation of the mean from at least 6 wells across 2 or 3 independent experiments. (f) $(\mathrm{i}-\mathrm{v})$ Plots showing the relative wavelength shift against time measured by biolayer interferometry of sensors coated with p24 to seven different concentrations of each analyte for (i) 59H10; (ii) 48G11 (raw data shown instead of relative binding as raw data showed no binding; therefore, data could not be normalized as other analytes); (iii) 37E7 nanobody; (iv) CPHIV-1/2; (v) NIH-3537. (g) Summary plot to compare the binding affinities of 59H10 (blue circles), CP-HIV-1/2 (red squares), NIH-3537 (green diamonds), and 37E7 (orange triangles). The log-linear plot shows the fractional saturation signal as a function of analyte concentration and the fits correspond to the Langmuir Adsorption Isotherm model. (h) A plot comparing the measured observed rate constants, $k_{\mathrm{obs}}$, for $59 \mathrm{H} 10$ (blue circles) and CP-HIV-1/2 (red squares) as a function of the analyte concentration. The linear regression fit shown was used to determine the kinetic on-rate constant $k_{\text {on }}$ (slope) and off-rate constant $k_{\text {off }}$ (intercept) where $R^{2}=0.99955$ for $59 \mathrm{H} 10$ and $R^{2}=0.99193$ for CP-HIV-1/2. A summary of the thermodynamic and kinetic parameters of nanobodies and mAbs is given in Table 1.

sensor technologies that can detect p24 in POC tests suitable for all settings.

Monoclonal antibodies (mAbs) have been the bedrock of many diagnostics for decades, and the diversity of the antibody repertoire generated by immunization of animals is unmatched; yet, the process yields a molecule with a largely unknown sequence and structure, batch to batch variation, ${ }^{12}$ that is sensitive to denaturation, ${ }^{13}$ is insufficiently pliable, and is often simply too large for today's nano and quantum diagnostic technologies, for example, Förster resonance energy transfer quantum dot biosensors ( 1 to $10 \mathrm{~nm}) \cdot{ }^{14,15}$ Future advances in diagnostics depend on ground-breaking technological developments occurring at the interface of biology, nanoscale physics, chemistry, and engineering, with the creation of novel sensing tools and systems that permit the detection of ultralow levels of biomarkers. $^{16}$

Nanobodies, which are recombinant antigen-binding domains from camelid antibodies, offer a number of significant advantages over conventional monoclonal and polyclonal antibodies. Conventional mammalian antibodies are composed of variable heavy and light chains, and diversity is generated by recombination and hypermutation. The llama antibody repertoire comprises approximately $70 \%$ conventional antibodies with $30 \%$ heavy-chain only antibody but still possesses a comprehensive repertoire. ${ }^{17-19}$ The physical properties and characteristics of the variable domains of these heavy chain-only antibodies (VHH) have been studied in terms of their physical properties, and characteristics include resistance to inactivation by heat or low $\mathrm{pH}$, binding in the presence of inhibitors, and high potency and specificity. ${ }^{20-24}$ The VHH can be cloned into vectors and produced in E. coli and yeast; thus, these are highly manipulable and scalable to produce large quantities. ${ }^{25}$ Nanobodies are around a tenth the size of a conventional antibody at $11-15 \mathrm{kDa}$, permitting them access to clefts and regions of the target protein for which a conventional antibody would be too big. ${ }^{14,26}$ To date, applications for nanobodies developed and reported have included imaging, therapeutic applications, and targeted inhibitors of enzymes or neutralizing antibodies, among others, ${ }^{23,27-30}$ and potential future applications have been recently reviewed. ${ }^{31}$

Maximizing the sensitivity of diagnostic tests involves overcoming research challenges such as elucidating how each constituent interacts with its partners, from biological sensor components to the signal measured by the sensor device. The characteristics of nanobodies facilitate these analyses as their sequence can be easily determined due to the formation of the antigen-binding site by a single polypeptide chain. Additionally, less computational processing power is required for modeling of tertiary structure and interactions with binding partners compared to larger molecules such as mAbs.

Herein, we report the development of novel nanobodies to detect HIV-1 p24. Lead candidates were selected on the basis of affinity, and their binding properties to different p24 subtypes were investigated, including those against a 50-member panel representing major circulating strains of HIV-1. The biophysical thermodynamics and kinetics of nanobody-p24 interactions were then studied by protein interaction detection methods and compared to those of two mAbs. We were able to obtain a crystal structure at $2.8 \AA$ of a high-affinity nanobody bound to p24. Despite near-identical primary amino acid sequences, two of the nanobodies had very different binding properties, and therefore, we used molecular dynamics simulations to unravel the underpinning contribution of electrostatics and hydrophobic forces. We believe this is the first published study to report the characterization of HIV-1 anti-p24 nanobodies that have potential for use as capture tools in fourth generation HIV-1 diagnostics. Here, we focus on HIV-1 because of the enormous unmet clinical need for better diagnostics, but the principles of our work can also be applied to a broad range of other disease areas.

\section{RESULTS AND DISCUSSION}

Immunization of two llamas (Lama glama) with HIV-1 p24 (subtypes B/C) (Figure 1a) generated a panel of nanobodies against p24 (Figure 1b,c). From the panel, five nanobodies were identified by affinity screening as lead candidates, of which three (37E7, 59H4, and 59H10) were taken for further investigation. They were initially assessed for their ability to detect a broad range of p24 subtypes by an indirect enzymelinked immunosorbent assay (ELISA) with seven purified recombinant $\mathrm{p} 24$ subtypes (HIV-1 Group M subtypes B, C, C, D, CRF01_AE, and Groups $\mathrm{N}$ and O, Figure 1d).

The results are summarized in Figure $1 \mathrm{~d}$ and Table S1. Two comparator mAbs were evaluated in parallel, CP-HIV-1/2 (a commercial antibody, green line) and NIH-3537 from the AIDS Reagent Program (red line). ${ }^{34}$ The ELISA data (Figure 1d) and derived affinity constants (Table S1) highlight that the nanobody 59H10 (blue line) is able to bind to all subtypes with 0.2 - to 17 -fold affinity compared to the best comparator $\mathrm{mAb}$ tested. A control nanobody to Bacteriophage p2 ORF18 protein, $\mathrm{LABS}^{33}$ (black line), was also included and showed no binding.

The ability of $59 \mathrm{H} 10$ to detect a much larger range of HIV-1 subtypes was tested next, as the number of p24 variants 
Table 1. Summary of the Thermodynamic Equilibrium Dissociation Constant Values, $K_{\mathrm{D}}(\mathrm{M})$, Kinetic On-Rate, $k_{\text {on }}\left(\mathrm{M}^{-1} \mathrm{~s}^{-1}\right)$, Kinetic Off-Rate, $k_{\text {off }}\left(\mathrm{s}^{-1}\right)$, As Measured by Biolayer Interferometry, and the Corresponding Calculated Gibbs Free Energies, $\Delta G_{\text {expt }}(\mathrm{kJ} / \mathrm{mol})$, for Different Nanobodies and $\mathrm{mAbs}$ Binding to $24\left(\text { where } \Delta G_{\text {expt }=}-\mathrm{RT} \ln \left(1 / K_{\mathrm{D}}\right)\right)^{a}$

\begin{tabular}{llllll} 
& \multicolumn{2}{c}{ nanobody } & & \multicolumn{2}{c}{$\mathrm{mAb}$} \\
\cline { 2 - 3 } \cline { 5 - 7 } biophysical parameters & $59 \mathrm{H} 10$ & $37 \mathrm{E} 7$ & $\mathrm{NIH}-3537$ & $\mathrm{CP}$ HIV-1/2 \\
\cline { 2 - 3 }$K_{\mathrm{D}}(\mathrm{M})$ & $6.9(3.9-9.8) \times 10^{-10}$ & $1.1(0.9-1.4) \times 10^{-7}$ & & $2.2(1.5-2.5) \times 10^{-8}$ & $1.6(1.1-2.0) \times 10^{-9}$ \\
$k_{\text {on }}\left(\mathrm{M}^{-1} \mathrm{~s}^{-1}\right)$ & $3.7(3.6-3.8) \times 10^{5}$ & $4.2(3.0-5.4) \times 10^{4}$ & & $2.1(1.6-2.7) \times 10^{3}$ & $4.5(3.7-5.4) \times 10^{4}$ \\
$k_{\text {off }}\left(\mathrm{s}^{-1}\right)$ & $2.9(1.9-3.9) \times 10^{-4}$ & $6.4(2.3-10.5) \times 10^{-3}$ & & $1.7(1.5-1.9) \times 10^{-4}$ & $2.0(1.0-2.9) \times 10^{-4}$ \\
$\Delta G_{\text {expt }}(\mathrm{kJ} / \mathrm{mol})$ & $-54.4(-55.8 /-53.5)$ & $-41.3(-41.8 /-40.7)$ & & $-47.0(-46.4 /-45.1)$ & $-52.2(-53.2 /-51.6)$
\end{tabular}

${ }^{a}$ Each biophysical parameter is shown with the corresponding $95 \%$ confidence interval indicated in brackets. The $K_{\mathrm{D}}$ was determined from fits of the experimental data, shown in Figure 1f, using the Langmuir Adsorption isotherm (1:1). The $k_{\text {on }}$ and $k_{\text {off }}$ were determined from the observed reaction rate, $k_{\mathrm{obs}}$ (Figure S3). All assays were carried out using HIV-1 subtype B p24 (AG6054). mAb = monoclonal antibody.

available as recombinant proteins is limited. The Viral Diversity Program panel (EQAPOL, Duke University, USA) contains a selection of 50 viral strains that reflects the diversity of strains found worldwide, spanning most common strains to rare isolates and unusual recombinants. ${ }^{35}$ A sandwich ELISA was created with 59H10 and CP-HIV-1/2, and results in Figure 1e show that $59 \mathrm{H} 10$ has consistently strong levels of binding with $48 / 50$ members of this panel. This indicates binding across all the strains tested irrespective of the intersubtype sequence differences, including subtype $C$, which is currently the source of most infections worldwide, with 5 representatives within the EQAPOL panel. ${ }^{36,37}$ One notable exception was subtype D, where variable levels of nanobody sensitivity were observed (Figure S1). These findings are in agreement with other studies involving subtype D strains. ${ }^{38}$ Nevertheless, these results highlight that $59 \mathrm{H} 10$ shows excellent binding to all the major circulating strains of HIV worldwide.

The ELISA studies provided an initial semiquantitative screen of lead nanobodies candidates; however, a potential limitation is that different labeling protocols were necessary for nanobodies and $\mathrm{mAbs}$, which could potentially influence the binding constants. Therefore, to better understand the biophysical performance characteristics of the nanobodies, the thermodynamics and kinetics of binding to p24 were studied using the label-free method of biolayer interferometry which requires no sample labeling of the nanobodies or mAbs and instead relies on optical detection of interference patterns when binding occurs to p24 coated probes over time. Additionally, a new nanobody mutant was created from 59H10, dubbed 48G11, in which a single F93D mutation in the CDR3 loop was made to change the charge environment of this region. Initial biolayer interferometry binding studies showed that this single change in 48G11 abolished all binding to p24 (Figure S2). A summary of parameters obtained from the biolayer interferometry data is shown in Table 1 and the raw data in Figure 1f. The processing methodology for the data is shown in Figure S3. Of all the mAbs and nanobodies tested, $59 \mathrm{H} 10$ showed the fastest kinetic-on rates $\left(k_{\text {on }}, 3.5 \times 10^{5} \mathrm{M}^{-1} \mathrm{~s}^{-1}\right)$ and was found to be 10 - to 100 -fold faster than mAb comparators. $59 \mathrm{H} 10$ also showed the strongest thermodynamic equilibrium dissociation binding constant of all ligands tested, at a $K_{\mathrm{D}}$ of $6.9 \times 10^{-10} \mathrm{M}$ making it by definition a high affinity antibody (where $K_{\mathrm{D}}<1 \times$ $\left.10^{-9} \mathrm{M}\right)$. The corresponding calculated Gibbs free energy, $\Delta G$, is $-54.4 \mathrm{~kJ} / \mathrm{mol}$, where $\Delta G_{\text {expt }=}-\mathrm{RT} \ln \left(1 / K_{\mathrm{D}}\right)$. By comparison, measured dissociation constants for $\mathrm{mAbs}$ were found to be between 2.3- to over 10-fold weaker. Both nanobodies and $\mathrm{mAbs}$ showed good agreement with a 1:1 Langmuir adsorption isotherm model, although it cannot be ruled out that mAbs could interact bivalently, particularly at high loading densities of p24.

In order to probe the molecular basis for the binding of the nanobodies to p24, the two lead nanobodies (59H10 and 37E7) and the artificial mutant $48 \mathrm{G} 11$ were further characterized. Sequencing of 37E7 and 59H10 revealed high levels of amino acid identity (111/114), differing only in 3 amino acids, (F55G, D56Y, and P57A, Kabat numbering ${ }^{39}$ ) in the complementarity determining region (CDR) loop 2 (Table 2 ). This is intriguing considering that their binding affinity to

Table 2. Comparison of Variations in Amino Acid Sequence in the CDR2 and CDR3 for Different Nanobodies ${ }^{a}$

\begin{tabular}{cccc} 
& & \multicolumn{2}{c}{$\begin{array}{c}\text { variance in amino acid } \\
\text { sequence }\end{array}$} \\
\cline { 3 - 4 } $\begin{array}{c}\text { nanobody } \\
\text { name }\end{array}$ & $\begin{array}{c}\text { relative } \begin{array}{c}\text { thermodynamic binding } \\
\text { affinity }\end{array} \\
\text { 59H10 }\end{array}$ & $\begin{array}{c}\text { CDR2 } \\
\text { loop }\end{array}$ & $\begin{array}{c}\text { CDR3 } \\
\text { loop }\end{array}$ \\
$48 \mathrm{G} 11$ & high & FDP & $\mathrm{F}$ \\
$37 \mathrm{E} 7$ & none & FDP & $\mathrm{D}$ \\
& intermediate & GYA & $\mathrm{F}$
\end{tabular}

${ }^{a}$ Shown are the substituted variations between each nanobody only. $59 \mathrm{H} 10$ exhibits the "best" binding to p24, 48G11 has no binding, and 37E7 has an intermediate level. Sequence comparison is shown for the variant $\mathrm{CDR} 2$ and $\mathrm{CDR} 3$ loops only; the rest of the structure including CDR1 and loop 4 regions are identical for all three nanobodies. 48G11 was generated by site-directed mutagenesis to the CDR3 loop. 37E7 is a CDR2-loop variant identified from the original repertoire library.

p24 differs by orders of magnitude and complies with observations that in nanobodies all three hypervariable regions (CDR1, -2, and -3) are involved in antigen binding (Figure $1 \mathrm{~d}, \mathrm{e}$, Table 1 , and refs 40-42). To ascertain the effects of these substitutions on the tertiary structure of the nanobodies, the p24-59H10 nanobody complex was crystallized and its structure solved by molecular replacement (Figure 2 and Table 3).

While solving the structure, it became apparent that the $\mathrm{N}$ terminal domain was missing in the crystal, probably due to proteolysis in the crystallization drop. The crystal structure reveals that the nanobody $59 \mathrm{H} 10$ employs its $\beta$-sheet and CDR1, -2 , and -3 to interact with the C-terminal domain of p24 in contact with the $2 \mathrm{C}$-terminal $\alpha$-helices 10 and 11 (Figure $2 \mathrm{a}, \mathrm{b})$. The interaction is stabilized by one salt bridge between $59 \mathrm{H} 10$ R50 and p24 E213 and four hydrogen bonds (Figure 2c). 59H10 N32 OD1 hydrogen bonds to p24 Q219 NE2, W37 NE1 to the carbonyl of p24 A204, and 59H10 R50 NH1 to the carbonyls of p24 L205 and P207. Additional hydrophobic contacts involve W37, F47, F93, A95, L96, and A101 of 59H10 


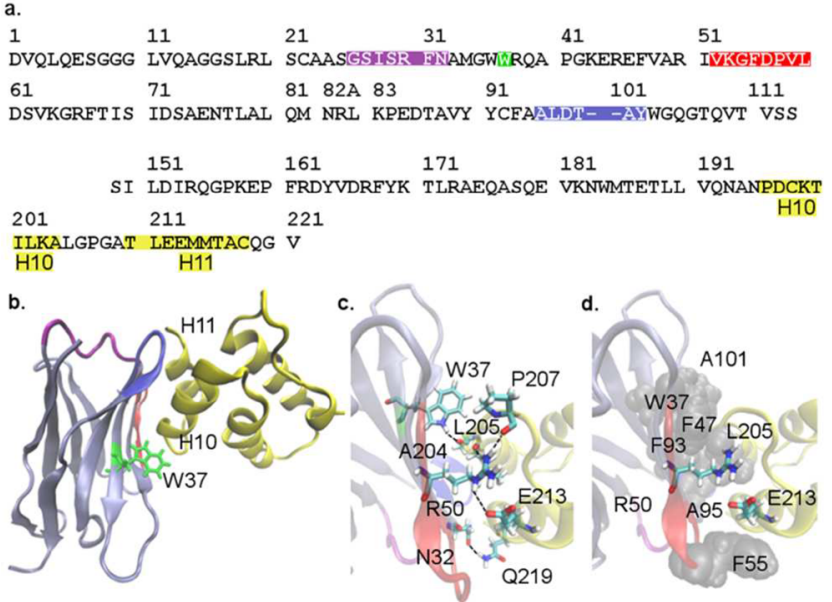

Figure 2. X-ray crystal structure of the epitope-paratope binding regions of p24 C-terminal domain in complex with the nanobody 59H10. (a) Sequence of the nanobody 59H10 and HIV capsid p24 present in the crystal structure. The different CDRs of the nanobody are highlighted, CDR1 (purple), CDR2 (red), and CDR3 (dark blue) along with 59H10 W37 (green) which is an unusual maturation mutation. The alpha helices $\mathrm{H} 10$ and $\mathrm{H} 11$ of p24, involved in the interaction with 59H10 nanobody, are highlighted in yellow. (b) Ribbon diagram of the paratope of $59 \mathrm{H} 10$ (light blue), a large interface involving CDR1, -2 , and -3 , and the epitope of p24 (yellow), involving helices 10 and $11.59 \mathrm{H} 10$ interacts with p24 helices 10 and 11. The different CDRs of the $59 \mathrm{H} 10$ are colored as described in (a). (c, d) Close up of the interactions. (c) salt bridges (59H10 R50 and p24 E213) and hydrogen bonds (59H10 W37 to p24 A204; 59H10 N32 to p24 Q219; 59H10 R50 to p24 P207 and L205) between interacting residues are indicated as dashed lines. (d) Visualization of the hydrophobic contacts around the 59H10 R50 to p24 E213 interaction, rendered in van der Waals and colored gray.

to p24 A204 and L205, the closest of these contacts to the 59H10 R50 and p24 E213 salt bridge. The HIV-1 capsid protein p24 exists in different oligomeric forms including dimers, pentamers, and hexamers. ${ }^{43}$ In principle, the nanobody $59 \mathrm{H} 10$ can potentially bind all forms (PDB code $5 \mathrm{HGN}){ }^{44}$ (PDB code 5I4T), ${ }^{45}$ (PDB code $\left.2 \mathrm{M} 8 \mathrm{~L}\right)^{46}$ ) due to the exposure of the binding epitope. Interaction with p24 present in the mature capsid ${ }^{47}$ would not be expected because the nanobody binding epitope is partly occluded in the polymeric form.

To validate the p24 epitope for $59 \mathrm{H} 10$, recombinant p24 with a truncation after L189 (cleaving helix 9) was used in an indirect ELISA, and the results were compared to a full p24. A range of conventional $\mathrm{mAbs}$ was used that had previously had their epitopes mapped, in addition to polyclonal antibodies. Figure S4 shows that while antibodies mapped to bind between helices 1 and 8 showed equivalent binding to full or truncated $\mathrm{p} 24,59 \mathrm{H} 10$ showed no binding to the truncated form of $\mathrm{p} 24$. This provides strongly supportive functional data for the epitope of p24 shown by the crystal data in Figure 2.

To unravel the molecular basis of the differences in affinity between the nanobodies and p24, caused by the single (48G11) or triple mutations (37E7) in the CDR3 or CDR2 loops, respectively, three-dimensional conformations of the selected nanobodies and their interactions with p24 were next investigated using molecular dynamics simulations. First, to ensure in silico models were created to be compatible with the crystal structure of $59 \mathrm{H} 10$ (Figure 2) and to probe the individual tertiary structures of the nanobodies individually,
Table 3. Crystallographic Data and Refinement Statistics ${ }^{a}$

\begin{tabular}{|c|c|}
\hline & p24-59H10 \\
\hline \multicolumn{2}{|l|}{ data collection } \\
\hline space group & $P 1211$ \\
\hline \multicolumn{2}{|l|}{ cell dimensions } \\
\hline$a, b, c(\AA)$ & $40.993,72.228,69.953$ \\
\hline$\alpha, \beta, \gamma(\mathrm{deg})$ & $90,91.398,90$ \\
\hline wavelength & 0.976251 \\
\hline resolution $(\AA)$ & $36.11-2.76(2.91-2.76)^{a}$ \\
\hline$R_{\text {merge }}$ & $0.147(0.712)$ \\
\hline$I / \sigma I$ & $6.1(1.7)$ \\
\hline completeness (\%) & $98(98.5)$ \\
\hline multiplicity & $3.0(3.0)$ \\
\hline \multicolumn{2}{|l|}{ refinement } \\
\hline resolution $(\AA)$ & 2.76 \\
\hline no. reflections & 10360 \\
\hline$R_{\text {work }} / R_{\text {free }}$ & $0.2211 / 0.2535$ \\
\hline \multicolumn{2}{|l|}{ no. atoms } \\
\hline protein & 2898 \\
\hline ligand/ion & 0 \\
\hline water & 0 \\
\hline \multicolumn{2}{|l|}{ B-factors } \\
\hline protein & 43.01 \\
\hline \multicolumn{2}{|l|}{ ligand/ion } \\
\hline \multicolumn{2}{|l|}{ water } \\
\hline \multicolumn{2}{|l|}{ RMS deviations } \\
\hline bond lengths $(\AA ̊)$ & 0.003 \\
\hline bond angles (deg) & 0.58 \\
\hline PDB code & $5 \mathrm{O} 2 \mathrm{U}$ \\
\hline
\end{tabular}

${ }^{a}$ Data from 1 crystal was used for the structure determination.

structural models were created by homology. These were referenced to the published nanobody D7 (PDB 2XA3), which has associated literature, ${ }^{40}$ to facilitate comparison to previous studies. D7 is a nanobody which binds to a different HIV antigen, the envelope protein gp120, and has a long, flexible CDR3 loop of 18 amino acids. ${ }^{40}$ To create starting structures for relaxation, the input for nanobody atomic coordinates was derived from structure homology using Swissprot. ${ }^{48}$ To obtain equilibrated and stable nanobody geometries, molecular dynamics simulations were run for $100 \mathrm{~ns}$ using an NVT ensemble at $333 \mathrm{~K}$. Figure 3a shows the crystal structure of PDB entry 2XA3, for nanobody D7, shown here for comparison to the p24 binding nanobodies. The mode of interaction for D7 binding is thought to be via the long CDR3 loop. The long CDR3 is found in another gp120-binding nanobody, A12 (PDB 3RJQ), and is thought to mediate a favorable inserted configuration into the pocket of gp $120 .{ }^{40}$ Its absence in the p24-binding nanobodies suggests that they could bind by a completely different mechanism.

Comparisons of structure and sequence are shown in the view of superimposed 59H10, 48G11, and 37E7 and colored by sequence homology in Figure $3 \mathrm{~b}$ and structural homology in Figure $3 \mathrm{c}$. Figure $3 \mathrm{~b}$ highlights the extensive (blue) homology with two regions of divergence. A comparison of Figure 3b,c shows that the sequence and structural differences do not exactly overlay. The structures of the nanobodies typically comprise an antiparallel barrel shape of $9 \beta$-strands connected by loops that make $2 \beta$-sheets. The exception is 37E7, which shows an $\alpha$-helix turn in the CDR1. This CDR1 $\alpha$-helix turn appears to be a secondary effect of changes in the CDR2 loop between $59 \mathrm{H} 10$ and $37 \mathrm{E} 7$. Figure $3 \mathrm{~d}-\mathrm{f}$ shows the equilibrated 


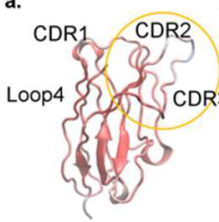

D7

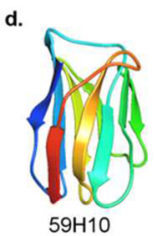

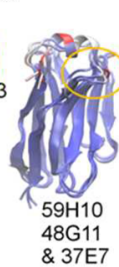
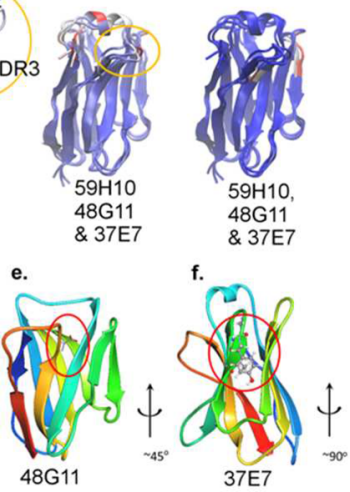

Figure 3. Examination of a measured crystal structure of nanobody D7 compared to calculated structures of 5910, 48G11, and 37E7 from molecular dynamics simulations. (a) Crystal structure of PDB entry 2XA3, nanobody D7, which binds HIV gp $120 .^{40}$ The yellow circle highlights the long CDR3 loop (in white), likely implicated in the gp120 binding interaction. (b) The minimized structures of the nanobodies reported here, with 37E7 and 48G11 superimposed onto $59 \mathrm{H} 10$, colored by amino acid sequence homology. (c) As in (b) but colored by structural homology. For both homology models, the blue indicates total conservation, red indicates no homology, and white is moderate conservation. The highlighted CDR3 loop (yellow circle) is much longer in the gp120-binding D7 as opposed to the p24-binding nanobodies. Structures of (d) 59H10; (e) 48G11; (f) 37E7; shown in rainbow coloring. The amino acid sites are shown as ball and stick where they differ from $59 \mathrm{H} 10$ and are highlighted in red circles. The orange loop is the CDR3 indicated for comparison with D7. Structures were equilibrated for $100 \mathrm{~ns}$.

molecular dynamics simulated structures of $59 \mathrm{H} 10$ (d), 48G11 (e), and 37E7 (f) separately to highlight the variant amino acids, drawn in ball-and-stick and circled in red. A comparison of the structure of $59 \mathrm{H} 10$ derived from the crystal (Figure 2) and from molecular dynamics atomistic simulations modeling $59 \mathrm{H} 10$ under experimental conditions used for the crystallization show that the root-mean-square deviation (RMSD) of the crystal and molecular dynamics structure after relaxation and equilibration is small, at $1.29 \AA$ (Figure S5). The RMSDs of all molecular dynamics structures (including 48G11 and 37E7) with time are shown in Figure S6. These show that, after relaxation and equilibration, structures do not deviate far from initial postequilibration coordinates. There is no upward drift shown over time, which indicates structural stability and that the models are suitable for aiding understanding of the differences in structure between the nanobodies.

The crystal structure of $59 \mathrm{H} 10$ and p 24 was used as a basis for molecular dynamics simulations of binding between $59 \mathrm{H} 10$, $48 \mathrm{G} 11$, or $37 \mathrm{E} 7$ and p24. This was done to probe the binding interaction in depth in bioassay conditions and to investigate the relative differences between the three nanobodies. The energetic contributions of individual residues were calculated, and models showing the importance of the residues at the interfaces are shown in Figure 4. The figures here show key amino acids colored red for favorable or blue for unfavorable contributions to binding. These total contributions are found from a Molecular Mechanics Poisson-Boltzmann Surface Area (MMPBSA) method and sum the electrostatic and van der Waals contributions (from the molecular dynamics) and the polar and apolar contributions (from the solution of the Poisson-Boltzmann equation and surface tension analysis,
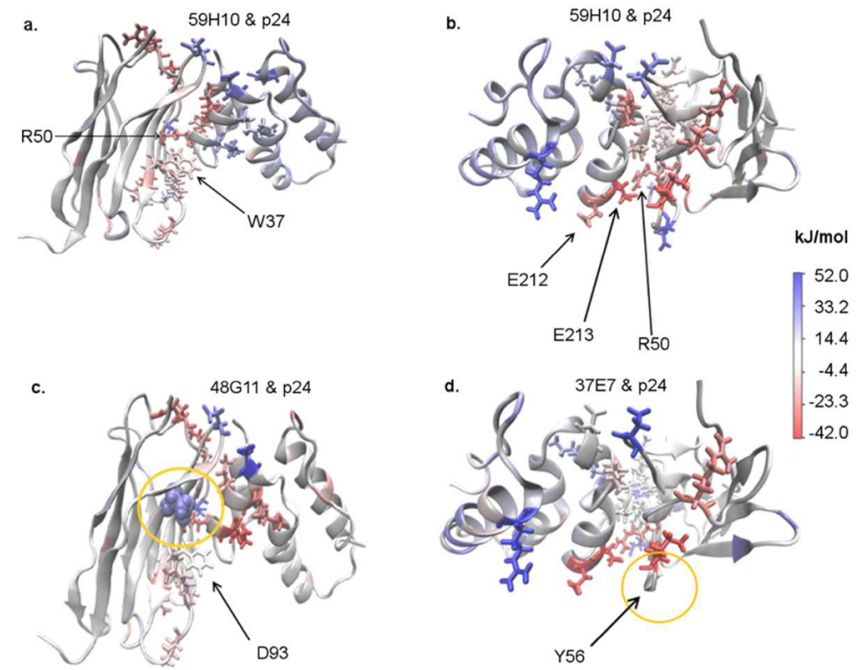

Figure 4. Analysis of molecular dynamics simulations to show the binding interfaces of nanobodies and p24, colored red for favorable or blue for unfavorable contribution to binding. (a) $59 \mathrm{H} 10$ and p24, shown in two orientations. The salt bridge formed between $59 \mathrm{H} 10$ R50 and p24 E213, as observed in the crystal (Figure 2), persists throughout the $40 \mathrm{~ns}$ dynamics as a highly energetically favorable interaction. The unusual maturation mutation, W37, also contributes favorably to the interface contacts; it forms a hydrogen bond and makes hydrophobic contacts with p24. (b) As in (a) but in a different orientation. A second glutamate (p24 E212) also contributes favorably to the binding. (c) 48G11 shown in the same orientation as $59 \mathrm{H} 10$ in (a). The mutation F93D is drawn in van der Waals render and clearly identifies as unfavorable. (d) 37E7 shown in the same orientation as $59 \mathrm{H} 10$ in (b). The substitution D56Y appears to eliminate an unfavorable charged interaction close to the 59H10-R50: p24 E213 salt bridge interaction, compare with the Y56 circled in yellow in (d).

respectively). From this analysis and a comparison of the three nanobodies, it appears that the 59H10 R50 and p24 E213 salt bridge is an important and very favorable interaction.

Figure S7 shows scatter plots for the numeric breakdown of the energetic contributions as visualized in Figure 4, individually for the nanobody and p24. The contributions made by each individual nanobody residue are highly homologous for 59H10, 48G11, and 37E7, differing only in the expected variant residues (D/Y56 and D/F 93, Figure S7a). The corresponding scatter plot for the energetic contribution of individual amino acids of p24 in Figure S7b, however, shows a greater level of variation for the three complexes of p24 when it is bound to $59 \mathrm{H} 10,48 \mathrm{G} 11$, or 37E7. For example, R162 of p24 in complex with 37E7, and D197 of p24 in complex with $48 \mathrm{G} 11$ both are significantly unfavorable to binding, in contrast to p24 in complex with 59H10, where R162 and D197 are slightly unfavorable or neutral, respectively. E212 and E213 of p24 in complex with $59 \mathrm{H} 10$ or $37 \mathrm{E} 7$, however, are both favorable interactions in comparison to $48 \mathrm{G} 11$, for which the interaction is slightly unfavorable. Notably, other interaction differences do not deviate from one another by much more than $\pm 10 \mathrm{~kJ} / \mathrm{mol}$ (approximately equivalent to a hydrogen bond), whereas residues with differences greater than this, as given in the above examples, are all charged amino acids. This demonstrates small changes to the amino acid composition in the three nanobodies has the consequence of disrupting the overall electrostatics of binding with respect to the same antigen with effects on many of the residues. The crystal structure in Figure 2 shows 59H10 bound to C-terminal helices 
10 and 11 ; however, Figure S7b shows that the effect of nanobody binding is not limited to a confined region of p24 and has a cascade effect over the rest of the protein.

To probe the importance of hydrogen bonds in binding, analysis of the 3 nanobodies each in complex with p24 was performed to determine hydrogen bond occupancy. This can change when the complex flexes and moves over time and in the presence of water molecules and salt ions. High occupancy indicates the frequency with which the hydrogen bond persists in the duration of the simulation as the thermodynamics of the interprotein contacts are monitored. The histogram in Figure 5 a shows the relative occupancy of each of the hydrogen bonds for those suggested by the crystal structure (Figure 2) and those between any of the three nanobodies and p24 that are occupied for at least $11 \%$ of the molecular dynamics simulation. The hydrogen bonding is defined by a suitable donor and acceptor atom being within a distance of $4 \AA$ and angle of $30^{\circ}$ (as suggested by the crystal structure). Of note are the high occupancy of the bonds between all nanobodies' R50 to p24 E213 and the complete absence of the hydrogen bond between 48G11 W37 and p24 A204. For 59H10, the key interactions are seen to be 59H10 R50 to p24 E213 (salt bridge, hydrogen bond), 59H10 W37 to p24 A204 (hydrophobic interaction, hydrogen bond), and 59H10 T98 to p24 D197 (hydrogen bond; T98 is in the CDR3). 59H10 R50 to p24 E213 is a persistent hydrogen bond and is also involved in formation of a salt bridge (see Figures 2 and 4). For the 37E7-p24 complex, fewer hydrogen bonds are formed by 37E7 R50 to p24 E213, as p24 E213 also shares intraprotein hydrogen bonds with p24 T210, drawing it away from a close interaction with 37E7 R50). A similar case is seen for $48 \mathrm{G} 11 \mathrm{R} 50$. The $48 \mathrm{G} 11 \mathrm{~W} 37$ to p24 A204 hydrogen bond is abolished entirely by the nearby F93D mutation (59H10 to 48G11) where the introduction of hydrophilic D disturbs an otherwise hydrophobic patch. The nanobody W37 to p24 A204 interaction is eliminated for $48 \mathrm{G} 11$, and the hydrogen bonding between $48 \mathrm{G} 11$ or $37 \mathrm{E} 7$ R50 and p24 E213 is less important. Note that p24 T210 shares hydrogen bonds in these cases with p24 E213, with the effect of weakening the nanobody R50 to p24 E213. Also shown in Figure 5a is p24 P207, which moves away from the hydrogen bonds found with $59 \mathrm{H} 10 \mathrm{R} 50$ in the crystalline form when in simulation in solution; hence, no occupancy is found.

The WXR motif (59H10 amino acids 36-38) is highly conserved, with the central $\mathrm{X}$ amino acid most commonly $\mathrm{F}, \mathrm{R}$, or a minority V. A sequence logo representing the relative distribution of amino acids found at this site is shown in Figure S8. An unusual "W" at position 37, giving WWR, was found in only two other unique sequences out of almost 800 available from the NCBI (GenBank AJ237346 and AF442913 ${ }^{49,50}$ ). This W37 was predicted both by analysis of the crystal structure and from molecular dynamics simulations to positively contribute to binding by formation of a hydrogen bond and to create an alignment of hydrophobic patches on 59H10 and p24, eliminated by F93D of 48G11.

The 59H10 R50 to p24 E213 interaction is clearly dominant (present in every molecular dynamics "snapshot") with an occupancy of over $100 \%$ for all nanobodies. This is possible, because there are multiple options for hydrogen bond pairs formed between the arginine and glutamate (Figure $5 \mathrm{~b}, \mathrm{c}$ ). A maximum of four interactions is possible at an optimal orientation of the amino acids, as observed in $59 \mathrm{H} 10$ but not the others (see inset to Figure 5b), indicating this combination of a strong electrostatic interaction from the salt bridge and
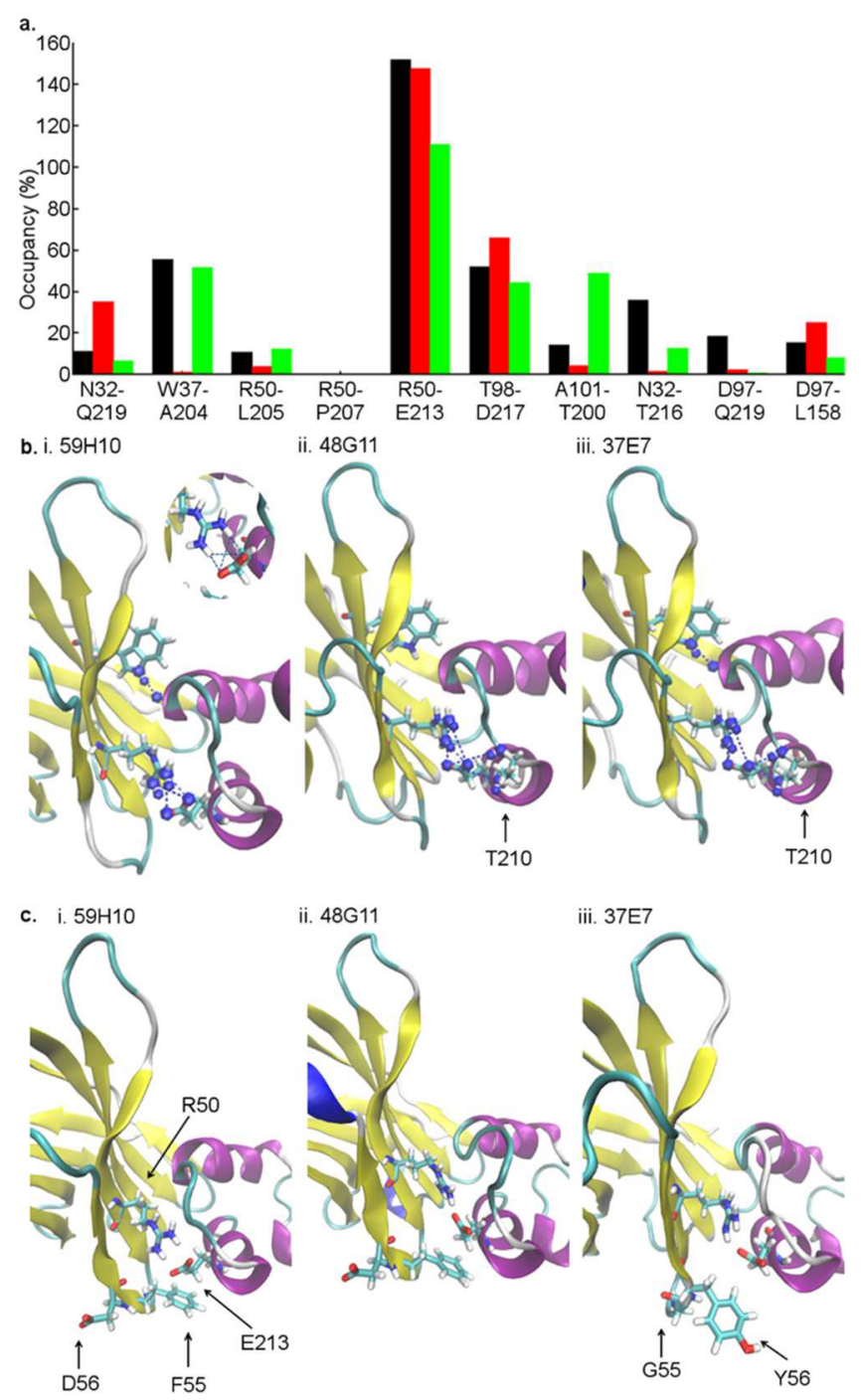

Figure 5. (a) Histogram to compare the occupancy of hydrogen bonds over the course of molecular dynamics simulations: black, $59 \mathrm{H} 10$; red, 48G11; green, 37E7. Examination of the occupancy of these bonds can indicate the relative importance of each interaction in stabilizing the complexes. Occupancy can reach over $100 \%$, indicating more than one hydrogen bond can form for the amino acid pair under consideration. (b) Visualization of hydrogen bond occupancy from the results of the molecular dynamics simulations using blue spheres on the atoms to indicate occupancy over the duration of the simulation. Shown are (i) 59H10, (ii) 48G11, and (iii) 37E7. Inset: representation of the four potential hydrogen bonds that can be formed between $59 \mathrm{H} 10 \mathrm{R} 50$ and p24 E213. This enables a stronger interaction between the 59H10 R50 to p24 E213 salt bridge, with the possibility of up to four hydrogen bonds forming at an optimal orientation. (c) Visualization of the nanobody R50 to p24 E213 maximal position in (i) 59H10, versus the suboptimal positioning in (ii) $48 \mathrm{G} 11$ and (iii) 37E7. Shown also are the F55G and D56Y mutations (59H10 to 37E7) because of their proximity to the salt bridge formed by nanobody R50 to p24 E213. It could be that there is an important network of charges provided by the F55-D56 that stabilizes this salt bridge further, perhaps a forming an anion $-\pi$-anion interaction between 59H10 D56 and 59H10 F55 and p24 E213.

multiple hydrogen bonds at an advantageous positioning of these amino acids results in a high affinity nanobody-p24 interaction. Figure $5 \mathrm{c}$ also shows that, although the substitution of D56Y (from 59H10 to 37E7) may appear to eliminate a potentially repulsive charge at the site of the salt bridge, a more 
accurate determination of the charge distribution and polarization effects is required where the geometry of amino acids of $59 \mathrm{H} 10$ from the molecular dynamics appears to suggest a favorable anion $-\pi$-anion interaction between $59 \mathrm{H} 10$ D56 and 59H10 F55 and p24 E213. This is not observed for 37E7.

To further probe the nature of the conjectured electrostatic interactions between p24 and 59H10, electrostatic potential energy maps of surfaces were calculated. Here, the Adaptive Poisson-Boltzmann Solver was used to solve the PoissonBoltzmann equation, and the PDB2PQR interfaced with UCSF Chimera using Assisted Model Building with Energy Refinement (AMBER) force fields for simulation of charge and atomic radii was used. ${ }^{51,52}$ Potential isocontours are colored for $+1 k T / e$ (blue) and $-1 k T / e$ (red) for all electrostatic potential maps shown in Figure 6. The electrostatic potential surfaces were calculated for all three nanobodies and found to show large variations in charge distributions that result from the single and triple amino acid substitutions. These are shown in order of positivity 48G11<59H10 < 37E7 in Figure 6a-c, respectively. Note that the changes in charge due to the substitutions affect the structures of the binding interface and vice versa. That is, the structure affects the distribution of charges, so that the binding surface complementarity is altered quite strikingly for these three nanobodies. The white skeleton structures are shown below the electrostatic potential maps to permit comparison of the binding interface of the three nanobody structures. These simulations reveal the dramatic effects that can accrue from minor mutations, with one or three amino acid changes out of 114 changing the spatial and electrostatic environment of the binding site. The ease with which single or multiple residues of these nanobodies can be mutated, and hence alter their functional interfaces, makes them particularly pliable as tools for nanoscale technology and in the design of highly efficient capture ligands for next generation HIV tests. As shown in Figure 6b, 59H10 shows an overall positively charged patch (circled in yellow) which corresponds to the binding interface. This has electrostatic complementarity to the binding interface of p24 (shown in Figure 6d) which is an overall negatively charged patch (circled in yellow). In comparison, 48G11 and 37E7 (Figure 6a,c, respectively) do not have a perfectly positive electrostatic region, demonstrating less compatibility with the electrostatic potential of the $\mathrm{p} 24$. As shown, small changes in amino acids, with large effects on charge, seem to define the relative binding behavior.

Given this electrostatic complementarity observed at the binding interfaces of the $59 \mathrm{H} 10$ and $\mathrm{p} 24$, it was of interest to compare subtypes of p24 that exhibit less strong binding. Though $59 \mathrm{H} 10$ binds well to a breadth of subtypes, there were exceptions found of some strains belonging to subtypes $\mathrm{C}$ (one of seven tested) and D (two of four tested; Figures 1d and S1). It has been reported that subtype $\mathrm{D}$ has been particularly hard to detect in point of care tests on the field. ${ }^{38}$ The electrostatic potential of the p24 C-terminal domain binding interfaces of strains that bound $59 \mathrm{H} 10$ variably are shown in Figure $6 \mathrm{f}-\mathrm{h}$. Figure $6 \mathrm{~g}, \mathrm{~h}$ shows disruption in the negative electrostatic potential face at the p24 C-terminal binding site, indicating electrostatic complementarity is an important contributor to strong binding. These two exceptions to the strong binding by $59 \mathrm{H} 10$ to other strains of these subtypes (Figure $1 \mathrm{~d}-\mathrm{f}$ ) support the hypothesis that, if there is disruption to the homogeneity of the negative electrostatic potential surface on the p24 binding interface or if there is a disruption to the homogeneity of the
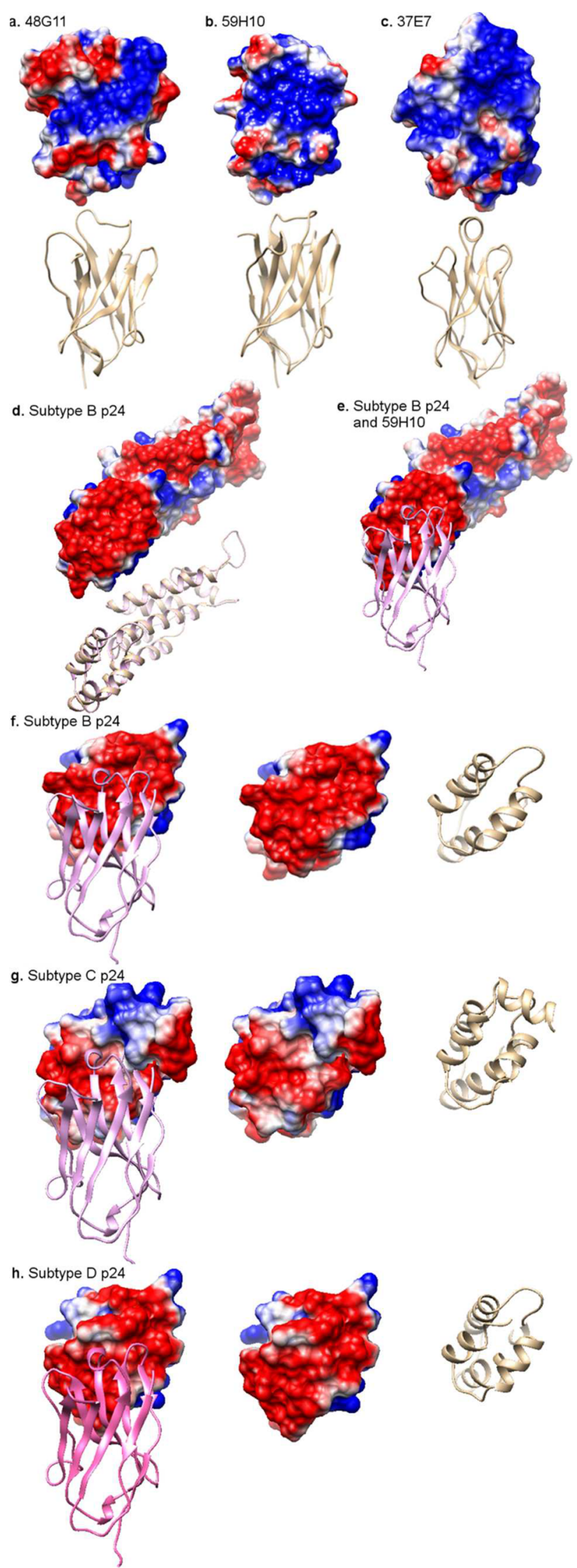

Figure 6. Electrostatic surface potentials of nanobodies and p24. Potential isocontours are colored for $+1 \mathrm{kT} / \mathrm{e}$ (blue, positive) and $-1 \mathrm{kT} / \mathrm{e}$ (red, negative). Electrostatic potentials and skeleton structures of the binding interface for (a) 48G11, (b) 59H10, and 
Figure 6. continued

(c) 37E7 are shown in order of overall positivity (37E7 has the highest total positive charge). Note the changes in amino acids at CDR2 and CDR3 create an electronegativity gradient over the entirety of the nanobodies from $48 \mathrm{G} 11$ (least electropositive), through $59 \mathrm{H} 10$ to 37E7 (most electropositive). Circled in yellow is the binding interface in $59 \mathrm{H} 10$ (b). (d) Electrostatic potentials and skeleton structures of full p24 (subtype B, based on PDB code 1E6J). The region circled in yellow highlights the binding interface for 59H10. The skeleton structure of p24 is shown below for comparison. (e) Electrostatic potentials of full p24 and skeleton structure of 59H10, showing 59H10 binding to p24. There is charge complementarity upon complex formation between the positive (blue region on the 59H10) and the negative (red region on the p24). (f) The electrostatic potential of the p24 binding site for $59 \mathrm{H} 10$ is shown for specific isolates of different subtypes of p24; (i) subtype B (as mentioned previously, for comparison), (ii) subtype C, ARP695, and (ii) subtype D, isolate DEMD10CM009.

positive electrostatic potential surface on the nanobody binding interface, there appears to be diminished binding. Disrupting the electrostatic complementarity at the p24 binding interface appears to adversely affect the binding kinetics, as demonstrated in the functional data (Figure 1f). We can conjecture that an overall electropositive map on the nanobody that complements and an overall electronegative map on the Cterminal of the p24 are preferable for strong affinity binding.

To conclude, this paper reports the isolation of a novel nanobody that binds to the earliest protein marker of HIV, p24, with high affinity, fast on-rates, and broad subtype specificity. We report an X-ray crystal structure, which shows the binding epitope and paratope regions on the p24 antigen 59H10 nanobody, respectively. Igonet et al. ${ }^{53}$ previously reported a nanobody that binds a different epitope region on p24 (PDB code 2XV6); however, the epitope reported and examined here would be accessible in many oligomeric forms of the p24 antigen. This is very advantageous and has particularly exciting repercussions in the design of a diagnostic or therapeutic tool. We have brought together functional experimental data, a crystal structure, and molecular dynamics simulations to elucidate the structural basis of this novel, high affinity nanobody. Further, the atomistic detail from the crystal structure of $59 \mathrm{H} 10$ provides a template with which to compare nanobodies with differing affinities using molecular dynamics simulations. The apparently simple mutation of a single amino acid within the CDR3 loop causes a cascade effect on electrostatic charge distribution across the façade of the nanobody, with a massive impact on binding affinity. Indeed, the small size of these nanobodies, together with the concomitant facility with which their single chain structure can be manufactured and modeled, means that structurefunction study findings can be fed back to iteratively improve the design for specific functions. Simulations shown here highlight the importance of the salt bridge of 59H10 R50 to p24 E213, the hydrogen bond of 59H10 W37 to p24 A204 and the alignment of hydrophobic patches, and the hydrogen bond 59H10 CDR3 T98 to p24 D197 and electrostatic complementary regions for high affinity binding. This information is valuable with respect to the design and development of tools that exploit the 59H10-p24 interaction. There is much scope for applications of this new understanding. The rapid on-rates and strong binding constants of 59H10 are advantageous in diagnostic assay formats, resulting in a fast time for results and high sensitivity, which is particularly important in the context of POC tests used in a primary healthcare setting. Moreover, effective anti-HIV tools for use in diagnostics, prevention of transmission, and therapy need to be able to bind to the wide array of global HIV-1 subtypes with high affinity, and 59H10 fulfils these criteria. The development of biheads, formed from two $59 \mathrm{H} 10$ nanobodies joined by a flexible linker, could further enhance sensitivity via multimeric complex formation with engineered tags to orient nanobodies for all sensing applications. $^{22,54}$ Once the interaction is understood, modifications to affect parameters such as, for example, binding affinity or attachment site to a surface, can be manipulated to create biological sensors of maximum sensitivity.

To close, we speculate that the potential of nanobodies goes beyond replacing traditional antibodies in conventional laboratory assays and could lead to entirely new classes of biosensor technologies based on advanced nano and quantum mechanical tools. These include ultrasensitive plasmonic ELISAs and quantum dot FRET assays which directly benefit from smaller nanobody capture coatings and reduced donoracceptor distance to innovative nanoswitches triggered by the effect of nanobody-p24 complex formation.

\section{METHODS}

Nanobody Generation. The library was produced from inoculation of two lama glama, and nanobodies were selected and produced in E. coli as described previously, ${ }^{29,55}$ using recombinant p24 immunogens (ARP678, ARP695, and EVA620) from the AIDS Regent Program (NIBSC, Potters Bar, UK).

Mutagenesis. For mutagenesis of $59 \mathrm{H} 10$ vector, oligonucleotides EG185 (GCCGTCTATTACTGTGATGCAGCCCTTGACACCGCCTACTGG) and EG186 (CCAGTAGGCGGTGTCAAGGGCTGCATCACAGTAATAGACGGC) were amplified with PCR using the following program and Pfu Turbo (Stratagene): $95{ }^{\circ} \mathrm{C}$ for $2 \mathrm{~min} ; 18$ cycles of $95{ }^{\circ} \mathrm{C}$ for 30 s, $55{ }^{\circ} \mathrm{C}$ for $1 \mathrm{~min}$, and $68{ }^{\circ} \mathrm{C}$ for $4 \mathrm{~min}$; $68{ }^{\circ} \mathrm{C}$ for $5 \mathrm{~min}$. Samples were digested with DpnI to remove template DNA, concentrated by $\mathrm{NaAc} / \mathrm{EtOH}$ precipitation, and used to transform E. coli HB101 (Promega). Clones were confirmed by sequencing. Vector was retransformed into TG1 (Lucigen) prior to expression.

Recombinant p24 Protein. The following were used in the study: AG6054 (B; BH10 Aalto BioReagents, Ireland), EVA620 (B; LAI, NIBSC), ARP0695 (C; consensus NIBSC), HIV 1033 (CRF01 AE, CM240, ImmunoDX, USA), and Subtypes D, N, and O (NDK, 06CM-U14296, BCF06; SinoBiological).

Purified p24 Protein. The bacterial expression plasmid pET11a, containing the HIV-1 capsid coding sequence, was obtained through the NIH AIDS Reagent Program, Division of AIDS, NIAID, NIH: pWISP98-85 from Dr. Wes Sundquist. The recombinant HIV-1 capsid protein p24 was purified as described previously. ${ }^{56}$ Purified p24 was complexed with the nanobody $59 \mathrm{H} 10$, and the complex was further purified by size exclusion chromatography on a Superdex 75 10/300 GL in a buffer containing $25 \mathrm{mM}$ Hepes, pH 7.5, $150 \mathrm{mM} \mathrm{NaCl}$.

Enzyme-Linked Immunosorbent Assay (ELISA). ELISA was used in both indirect and sandwich format to assay for activity. For indirect ELISAs, recombinant p24 was bound to MaxiSorp 96-well plates (Nunc) between 16 and $1000 \mathrm{ng} / \mathrm{mL}$ in $50 \mathrm{mM}$ sodium carbonate buffer $\mathrm{pH} 9.6$ at $4{ }^{\circ} \mathrm{C}$ overnight. The following morning, plates were washed and blocked in $2 \%$ 
BSA or nonfat milk in PBS-0.05\% Tween for $2 \mathrm{~h}$. Nanobody or $\mathrm{mAb}$ (NIH-3537, AIDS Reagent Program; CP-HIV-1/2, Capricorn Products) was added in blocking buffer for $2 \mathrm{~h}$. After binding of primary antibody, plates were washed $4 X$ in PBS-T; then, secondary antibody was bound (goat antimouseHRP, Dako; antimyc-HRP, Roche; streptavidin-HRP, Sigma) for $1-2 \mathrm{~h}$. Plates were washed $4-6 \times$ in PBS-T; peroxidase activity was assayed with $1 \times \mathrm{TMB}$ (eBioscience), and 5-15 min later, $0.2 \mathrm{M} \mathrm{H}_{2} \mathrm{SO}_{4}$ was added. The $\mathrm{OD}$ was read at 450 nm (SpectraMax, Molecular Devices). For sandwich ELISAs, assays were carried out essentially as above except capture antibodies were used to coat plates overnight at $78-313 \mathrm{ng} /$ $\mathrm{mL}$, and antigen was bound for $1.5 \mathrm{~h}$ at $37^{\circ} \mathrm{C}$.

Crystallization, Data Collection, and Structure Determination. Crystal screening was performed at the High Throughput Crystallization Laboratory (HTX lab, Grenoble) in 96-well sitting drop vapor diffusion plates (Greiner). Following optimization, crystals used for diffraction studies were grown at $20{ }^{\circ} \mathrm{C}$ in hanging drop vapor diffusion plates and obtained by streak seeding.

The p24-59H10 complex was crystallized in $100 \mathrm{mM}$ Tris base, $\mathrm{pH}$ 8.0, 20\%, and polyethylene glycol (PEG) 6000 by mixing $1 \mu \mathrm{L}$ of the reservoir and $1 \mu \mathrm{L}$ of protein at a concentration of $8 \mathrm{mg} / \mathrm{mL}$. The crystals were cryoprotected in the crystallization buffer containing $25 \%$ ethylene glycol and flash cooled in liquid $\mathrm{N}_{2}$ before data collection. Data were collected on beamline ID30-B at the ESRF at a wavelength of $0.976251 \AA$ A. Data were processed with the program XDS. ${ }^{57}$ The crystals belong to space group P1211 (Table 3).

The structure was solved by molecular replacement using the program Phaser ${ }^{58}$ using the PDB 2GOL (for p24) and 4LDO (for the nanobody 59H10) as input models. The model was rebuilt using $\operatorname{COOT}^{59}$ and refined using REFMAC ${ }^{60}$ and Phenix. ${ }^{61}$ Statistics for data reduction and structure refinement are presented in Table 3 . The models were evaluated by using COOT and Phenix validation tools. Two copies of the p24$59 \mathrm{H} 10$ complex are present in the asymmetric unit. Only the C-terminal domain of p24 is present in the crystal and chains A and $\mathrm{C}$ contain residues 149 to 221 . For the nanobody 59H10 and chains $\mathrm{B}$ and $\mathrm{D}$, residues are numbered according to Kabat. The structure was refined against $2.76 \AA$ data to an $R / R_{\text {free }}$ of $22.11 / 25.35 \% .98 .64 / 1.36 \%$ of the residues are within the most favored and allowed regions of a Ramachandran plot. Molecular graphics figures were generated with PyMOL (W. Delano; The PyMOL Molecular Graphics System, Version 1.8 Schrödinger, LLC, http://www.pymol.org).

Molecular Dynamics Simulations. All atomistic classical molecular dynamics (MD) simulations were performed using NAMD $^{62}$ and GROMACS packages ${ }^{63}$ and visualized using $\mathrm{VMD}^{64}$ and Chimera. ${ }^{51}$ Initial MD runs took models for the 48G11, 59H10, and 37E7 obtained from Swissprot. These structures were minimized, using the NAMD conjugate gradient and line search algorithm; relaxed geometry results are as shown in Figure 3b,c. Subsequently, these structures were prepared for a 100 ns NVT (canonical; constant volume, constant temperature) ensemble with Langevin dynamics in an explicit water sphere just covering the protein with no periodic boundary conditions. The TIP3P potential was used for the water interactions. Force fields par_all27_prot_lipid_na and top all27 prot lipid na.inp were used for the nanobodies. The simulations were run at $333 \mathrm{~K}$, with a Langevin damping coefficient of $1 \mathrm{ps}^{-1}$, in order to accelerate any possible observation of protein unfolding and probe conformational space. The final equilibrated structures from this run were used as input for production stage runs; see Figure $3 \mathrm{~d}-\mathrm{f}$ for skeleton models of the resulting conformations. These were put into cubic water boxes with $10 \AA$ between the box boundary and the edge of the protein. The production runs maintained a constant pressure of 1 atm at $310 \mathrm{~K}, \mathrm{NPT}$ (isothermal-isobaric) ensemble, and $\mathrm{pH} 7$ (approximately physiological $\mathrm{pH}$ ) where the disulfide bridge is intact. Full system periodic electrostatics were calculated using Particle Mesh Ewald (PME) grids with counterions added for charge neutrality. A cutoff of $12 \AA$ was used for the Lennard-Jones force field parameters; the SHAKE algorithm was used to constrain all hydrogen-containing bonds, and the time step was $2 \mathrm{fs}$, as in the equilibration step.

The MD simulations described above demonstrated the stability of the three nanobodies based on models. The experimental crystal structure of $59 \mathrm{H} 10$ bound to p24 also provided atomistic coordinates for the complex. This (PDB $5 \mathrm{O} 2 \mathrm{U}$ ) was then used as input for molecular dynamics (GROMACS) calculations to assess binding, ${ }^{63}$ with $48 \mathrm{G} 11$ and $37 \mathrm{E} 7$ superimposed onto the binding site for $59 \mathrm{H} 10$. For these binding comparisons, coordinate and topology files were parametrized using the AMBER99SB force field, with TIP3P water molecules added to solvate, surrounding the protein complex in the center of a dodecahedron periodic box with a side of at least $10 \AA$ between the edge of the box and the protein. Hydrogen atoms were added to imitate protonation states at $\mathrm{pH} 7$ and with a disulfide bridge accounted for in the nanobody and in the p24. In each case, the system was neutralized and $75 \mathrm{mM}$ of $\mathrm{NaCl}$ was added to match the X-ray conditions, and again, full system periodic electrostatics were calculated using Particle Mesh Ewald (PME). The minimization was performed using the steepest descent algorithm, and then, an equilibration was run using an NVT ensemble to heat to $310 \mathrm{~K}$ using a modified Berendsen thermostat; then, an NPT was run with Parrinello-Rahman coupling for pressure control at $1 \mathrm{~atm}$. Production runs were then completed for $40 \mathrm{~ns}$ total using a 2 fs time step, requiring 20 million steps of integration. All bonds were constrained with the parallel LINCS method, with short-range nonbonded electrostatic interactions calculated with a cutoff of $9 \AA$ and van der Waals with a cutoff of 1.4 $\AA$. This method follows the protocol outlined as in "g_mmpbsa", a GROMACS tool to calculate Molecular Mechanics Poission-Boltzmann Surface Area (MMPBSA) of binding interaction energies. ${ }^{65}$ Input into g_mmpbsa was then generated from the MD results: 400 frames every $0.1 \mathrm{~ns}$ was used to represent uncorrelated snapshots from the $40 \mathrm{~ns}$ duration. Energies from the molecular mechanics were calculated using the Lennard-Jones and Coulomb potential. Polar energies were calculated at an ionic strength of $\mathrm{NaCl} 75$ $\mathrm{mM}$, with the protein and solvent dielectric constants of 2 and 80 , respectively. As these systems are charged (with subtype B p24 at -4 electrons), the nonlinear Poisson-Boltzmann equation was solved using APBS. The apolar energies were calculated on the basis of a fitting to the surface tension for the solvent accessible surface area. Results of these simulations are displayed in Figures 4, 5 (MD), S6 (MD), and S7 (MMPBSA) and Table S2 (MMPBSA).

Biolayer Interferometry. A binding comparison of the nanobodies and mAbs (termed analytes) to immobilized p24 subtype B (AG6054) was performed on an Octet RED96 (FortéBio, UK). AG6054 was immobilized on amine-reactive probes (AR2G Biosensor, FortéBio). The probes were activated by a $5 \mathrm{~min}$ incubation in a freshly mixed sulfo-NHS-EDC 
solution $(10 \mathrm{mM} / 20 \mathrm{mM})$, before incubation for $10 \mathrm{~min}$ in a $20 \mu \mathrm{g} / \mathrm{mL}$ solution of AG6054 in $10 \mathrm{mM}$ acetate buffer (pH5) and then $5 \mathrm{~min}$ in a $1 \mathrm{M}$ solution of ethanolamine- $\mathrm{HCl}(\mathrm{pH}$ 8.5 ), washed in the kinetics buffer provided, and then dipped in the sample diluted in kinetics buffer. Each run consisted of testing seven different concentrations of analyte once. Analyte binding proceeded for at least $60 \mathrm{~min}$. Normalization was carried out using an in situ probe functionalized with p24 and dipped in sample solution without added analyte, to account for nonspecific reactions occurring on the surface. At high concentrations of analyte, the signal appears to plateau and reach equilibrium within the time frame of the experiment. However, at low analyte concentrations, the signal does not reach equilibrium within this time frame. Therefore, the equilibrium signal was determined by fitting the raw data for each concentration to the exponential equation: $y=A[1-$ $\left.\exp \left(-k_{\text {obs }} t\right)\right]$, where $y$ is the binding in $\mathrm{nm}, A$ represents the equilibrium signal, $k_{\mathrm{obs}}$ is the observed rate constant, and $t$ is time. The equilibrium signal $(A)$ as a function of the concentration was then fitted to a Langmuir isotherm $y=$ $B\left[c / K_{D}+c\right]$, where $K_{D}$ represents the binding affinity of the protein to $\mathrm{p} 24, B$ is the saturation signal when all the available sites are occupied, and $c$ is analyte concentration. The analyses in Figure $1 \mathrm{f}-\mathrm{h}$ were obtained by normalizing the raw data by the saturation signal $B$, following the method reported by Martin and co-workers. ${ }^{6,67}$ Figure $1 \mathrm{~g}$ is a $\log /$ linear plot of the fractional saturation (ratio $A / B$ ) as a function of the concentration, for each analyte. A detailed description of the analysis is provided in Figure S3. The kinetic on-rate constant $k_{\text {on }}$ and off-rate constant $k_{\text {off }}$ presented in Table 1 are defined by the equation $k_{\text {obs }}=k_{\text {off }}+k_{\text {on }} \times c$ and were determined as the slope and intercept, respectively, of the linear regression performed on $k_{\mathrm{obs}}$ as a function of the concentration (example shown for 59H10 and CP-HIV-1/2 in Figure S3). All data in Table 1 are presented with the corresponding $95 \%$ confidence intervals.

\section{ASSOCIATED CONTENT}

\section{S Supporting Information}

The Supporting Information is available free of charge on the ACS Publications website at DOI: 10.1021/acsinfecdis.6b00189.

Supplementary methods; binding data subtype D strains; biolayer interferometry binding data; processing of biolayer interferometry fractional saturation analysis; binding to a truncated p 24 by $59 \mathrm{H} 10, \mathrm{mAbs}$ and pAbs; superimposed crystal $59 \mathrm{H} 10 / \mathrm{p} 24$ and simulated $59 \mathrm{H} 10 /$ p24; RMSD plots; energetic contribution scatter plots; sequence logo; affinity constants calculated from ELISA data; binding energy contributions (PDF)

\section{AUTHOR INFORMATION}

\section{Corresponding Author}

*E-mail: r.a.mckendry@ucl.ac.uk.

\section{ORCID}

Eleanor R. Gray: 0000-0002-6515-839X

Rachel A. McKendry: 0000-0003-2018-6829

\section{Present Address}

${ }^{\text {II }}$ L.A.G.: Department of Infectious Diseases, King's College London, London SE1 9RT, UK.

\section{Author Contributions}

${ }^{\perp}$ E.R.G., J.C.B., and C.C. are joint first authors. J.C.B., E.R.G., R.A.M., B.L.J.W., and R.A.W. conceived the study. M.E.K. and C.T.V. generated the nanobodies. J.C.B., C.C., L.A.G., E.R.G., L.E.M., V.T., and B.L.J.W. performed the experiments. J.C.B., C.C., E.R.G., L.E.M., B.S.M., and B.L.J.W. analyzed the data. J.C.B., E.R.G., L.E.M., R.A.M., V.T., and R.A.W. discussed the implications. J.C.B., C.C., D.M.D., E.R.G., L.E.M., R.A.M., C.T.V., B.L.J.W., W.W., and R.A.W. wrote and revised the manuscript.

\section{Notes}

This paper presents independent research funded by the National Institute for Health Research (NIHR). The views expressed are those of the authors and not necessarily those of the NHS, the NIHR, or the Department of Health.

The authors declare the following competing financial interest(s): M.E.K. and C.T.V. are employed by a commercial company, QVQ B.V. This does not alter their adherence to policies on data sharing and materials. A studentship to V.T. was part-funded by OJ-Bio. The remaining authors declare that no competing financial interest exists.

\section{ACKNOWLEDGMENTS}

This work was funded by grants i-sense EPSRC IRC Early Warning Sensing Systems in Infectious Disease (EP/K031953/ 1) to E.R.G., J.C.B., V.T., R.A.W., and R.A.M. (www.i-sense.org. uk); EPSRC Grand Challenge in Healthcare Diagnostics: Multimarker Nanosensors for HIV (EP/G062064/1) to B.L.J.W. and R.A.M.; NIHR II-LA-1111-20004 Next generation mobile HIV diagnostics with wireless connectivity to E.R.G., J.C.B., V.T., R.A.W. and R.A.M.; Medical Research Council Grant G0801176 to L.E.M., L.A.G., and R.A.W.; Royal Society Wolfson Research Merit Award to R.A.M. W.W. is a member of the Institut Universitaire de France and acknowledges the platforms of the Grenoble Instruct Center (ISBG; UMS 3518 CNRS-CEA-UJF-EMBL) supported by the French Infrastructure for Integrated Structural Biology Initiative FRISBI (ANR-10-INSB-05-02) and GRAL (ANR-10-LABX-49-01) within the Grenoble Partnership for Structural Biology (PSB). We acknowledge and credit the developers of NAMD at the Theoretical and Computational Biophysics Group in the Beckman Institute for Advanced Science and Technology at the University of Illinois at Urbana-Champaign. We thank Dr. Morag A. Lewis for the llama drawing. We also thank the ESRF-EMBL Joint Structural Biology Group for access and support at the ESRF beamlines and J. Marquez (EMBL) from the crystallization platform.

\section{ABBREVIATIONS}

AMBER, assisted model building with energy refinement; APBS, adaptive Poisson-Boltzmann solver; CDR, complementarity determining region; ELISA, enzyme-linked immunosorbent assay; FRET, Förster resonance energy transfer; $\mathrm{mAb}$, monoclonal antibody; HIV, human immunodeficiency virus; $\mathrm{MD}$, molecular dynamics; MMPBSA, molecular mechanics Poisson-Boltzmann surface area; PDB, the Protein Data Bank; POC, point of care; $\mathrm{VHH}$, variable domains of heavy chain-only antibodies 


\section{REFERENCES}

(1) Rambaut, A., Posada, D., Crandall, K. A., and Holmes, E. C. (2004) The causes and consequences of HIV evolution. Nat. Rev. Genet. 5 (1), 52-61.

(2) UNAIDS. (2013) Global Report 2013, UNAIDS/JC2502/1/ EDOI, pp 1-198, UNAIDS, Geneva.

(3) Aghaizu, A., Brown, A. E., Nardone, A., Gill, O. N., and Delpech, V. C. (2013) HIV in the United Kingdom: 2013 Report, 1-39.

(4) Beck, E. J., Mandalia, S., Sangha, R., Sharott, P., Youle, M., Baily, G., Brettle, R., Gompels, M., Johnson, M., McCarron, B., et al. (2011) The cost-effectiveness of early access to HIV services and starting cART in the UK 1996-2008. PLoS One 6 (12), e27830.

(5) Cohen, M. S., Chen, Y. Q., McCauley, M., Gamble, T., Hosseinipour, M. C., Kumarasamy, N., Hakim, J. G., Kumwenda, J., Grinsztejn, B., Pilotto, J. H. S., et al. (2011) Prevention of HIV-1 infection with early antiretroviral therapy. N. Engl. J. Med. 365 (6), 493-505.

(6) Nosyk, B., and Montaner, J. S. G. (2012) The Evolving Landscape of the Economics of HIV Treatment and Prevention. PLoS Med. 9 (2), e1001174-2.

(7) Department of Health. (2011) Government Response to the House of Lords, Report of Session 2010-12: No vaccine, no cure: HIV and AIDS in the United Kingdom, pp 1-36, Crown, London.

(8) Youngs, J., and Hooper, C. (2015) Ethical implications of HIV self-testing. J. Med. Ethics 41 (10), 809-813.

(9) Busch, M. P., and Satten, G. A. (1997) Time course of viremia and antibody seroconversion following human immunodeficiency virus exposure. Am. J. Med. 102 (5B), 117-124.

(10) Faraoni, S., Rocchetti, A., Gotta, F., Ruggiero, T., Orofino, G., Bonora, S., and Ghisetti, V. (2013) Evaluation of a rapid antigen and antibody combination test in acute HIV infection. J. Clin. Virol. 57 (1), 84-87.

(11) Albertini, A., Lee, E., Coulibaly, S. O., Sleshi, M., Faye, B., Mationg, M. L., Ouedraogo, K., Tsadik, A. G., Feleke, S. M., Diallo, I., et al. (2012) Malaria rapid diagnostic test transport and storage conditions in Burkina Faso, Senegal, Ethiopia and the Philippines. Malar. J. 11 (1), 406.

(12) Baker, M. (2015) Reproducibility crisis: Blame it on the antibodies. Nature 521, 274-276.

(13) Vermeer, A. W., and Norde, W. (2000) The thermal stability of immunoglobulin: unfolding and aggregation of a multi-domain protein. Biophys. J. 78 (1), 394-404.

(14) De Genst, E., Silence, K., Decanniere, K., Conrath, K., Loris, R., Kinne, J., Muyldermans, S., and Wyns, L. (2006) Molecular basis for the preferential cleft recognition by dromedary heavy-chain antibodies. Proc. Natl. Acad. Sci. U. S. A. 103 (12), 4586-4591.

(15) Wegner, K. D., Lindén, S., Jin, Z., Jennings, T. L., Khoulati, R. E., van Bergen en Henegouwen, P. M. P., and Hildebrandt, N. (2014) Nanobodies and Nanocrystals: Highly Sensitive Quantum Dot-Based Homogeneous FRET Immunoassay for Serum-Based EGFR Detection. Small 10 (4), 734-740.

(16) de la Rica, R, and Stevens, M. M. (2012) Plasmonic ELISA for the ultrasensitive detection of disease biomarkers with the naked eye. Nat. Nanotechnol. 7 (12), 821-824.

(17) Hamers-Casterman, C., Atarhouch, T., Muyldermans, S., Robinson, G., Hammers, C., Songa, E. B., Bendahman, N., and Hammers, R. (1993) Naturally occurring antibodies devoid of light chains. Nature 363 (6428), 446-448.

(18) Tonegawa, S. (1983) Somatic generation of antibody diversity. Nature 302 (5909), 575-581.

(19) Muyldermans, S. (2001) Single domain camel antibodies: current status. Rev. Mol. Biotechnol. 74 (4), 277-302.

(20) van der Linden, R. H., Frenken, L. G., de Geus, B., Harmsen, M. M., Ruuls, R. C., Stok, W., de Ron, L., Wilson, S., Davis, P., and Verrips, C. T. (1999) Comparison of physical chemical properties of llama VHH antibody fragments and mouse monoclonal antibodies. Biochim. Biophys. Acta, Protein Struct. Mol. Enzymol. 1431 (1), 37-46.

(21) Gorlani, A., Brouwers, J., McConville, C., van der Bijl, P., Malcolm, K., Augustijns, P., Quigley, A. F., Weiss, R., De Haard, H., and Verrips, T. (2012) Llama antibody fragments have good potential for application as HIV type 1 topical microbicides. AIDS Res. Hum. Retroviruses 28 (2), 198-205.

(22) Els Conrath, K., Lauwereys, M., Wyns, L., and Muyldermans, S. (2001) Camel single-domain antibodies as modular building units in bispecific and bivalent antibody constructs. J. Biol. Chem. 276 (10), $7346-7350$.

(23) Lauwereys, M., Arbabi Ghahroudi, M., Desmyter, A., Kinne, J., Hölzer, W., De Genst, E., Wyns, L., and Muyldermans, S. (1998) Potent enzyme inhibitors derived from dromedary heavy-chain antibodies. EMBO journal 17 (13), 3512-3520.

(24) Abbady, A. Q., Al-Daoude, A., Al-Mariri, A., Zarkawi, M., and Muyldermans, S. (2012) Chaperonin GroEL a Brucella immunodominant antigen identified using Nanobody and MALDI-TOF-MS technologies. Vet. Immunol. Immunopathol. 146 (3-4), 254-263.

(25) van de Laar, T., Visser, C., Holster, M., López, C. G., Kreuning, D., Sierkstra, L., Lindner, N., and Verrips, T. (2007) Increased heterologous protein production by Saccharomyces cerevisiae growing on ethanol as sole carbon source. Biotechnol. Bioeng. 96 (3), 483-494.

(26) Koide, A., Tereshko, V., Uysal, S., Margalef, K., Kossiakoff, A. A., and Koide, S. (2007) Exploring the Capacity of Minimalist Protein Interfaces: Interface Energetics and Affinity Maturation to Picomolar KD of a Single-domain Antibody with a Flat Paratope. J. Mol. Biol. 373 (4), 941-953.

(27) Braun, M. B., Traenkle, B., Koch, P. A., Emele, F., Weiss, F., Poetz, O., Stehle, T., and Rothbauer, U. (2016) Peptides in headlock-a novel high-affinity and versatile peptide-binding nanobody for proteomics and microscopy. Sci. Rep. 6, 19211.

(28) Schepens, B., Ibanez, L. I., De Baets, S., Hultberg, A., Bogaert, P., De Bleser, P., Vervalle, F., Verrips, T., Melero, J., Vandevelde, W., et al. (2011) Nanobodies(R) Specific for Respiratory Syncytial Virus Fusion Protein Protect Against Infection by Inhibition of Fusion. J. Infect. Dis. 204 (11), 1692-1701.

(29) McCoy, L. E., Quigley, A. F., Strokappe, N. M., Bulmer-Thomas, B., Seaman, M. S., Mortier, D., Rutten, L., Chander, N., Edwards, C. J., Ketteler, R., et al. (2012) Potent and broad neutralization of HIV-1 by a llama antibody elicited by immunization. J. Exp. Med. 209 (6), 10911103.

(30) Kirchhofer, A., Helma, J., Schmidthals, K., Frauer, C., Cui, S., Karcher, A., Pellis, M., Muyldermans, S., Casas-Delucchi, C. S., Cardoso, M. C., et al. (2010) Modulation of protein properties in living cells using nanobodies. Nat. Struct. Mol. Biol. 17 (1), 133-138.

(31) Hassanzadeh-Ghassabeh, G., Devoogdt, N., De Pauw, P., Vincke, C., and Muyldermans, S. (2013) Nanobodies and their potential applications. Nanomedicine (London, U. K.) 8 (6), 10131026.

(32) Pardon, E., Laeremans, T., Triest, S., Rasmussen, S. G. F., Wohlkönig, A., Ruf, A., Muyldermans, S., Hol, W. G. J., Kobilka, B. K., and Steyaert, J. (2014) A general protocol for the generation of Nanobodies for structural biology. Nat. Protoc. 9 (3), 674-693.

(33) De Haard, H. J. W., Bezemer, S., Ledeboer, A. M., Müller, W. H., Boender, P. J., Moineau, S., Coppelmans, M.-C., Verkleij, A. J., Frenken, L. G. J., and Verrips, C. T. (2005) Llama antibodies against a lactococcal protein located at the tip of the phage tail prevent phage infection. J. Bacteriol. 187 (13), 4531-4541.

(34) Chesebro, B., Wehrly, K., Nishio, J., and Perryman, S. (1992) Macrophage-tropic human immunodeficiency virus isolates from different patients exhibit unusual V3 envelope sequence homogeneity in comparison with T-cell-tropic isolates: definition of critical amino acids involved in cell tropism. J. Virol. 66 (11), 6547-6554.

(35) Sanchez, A. M., DeMarco, C. T., Hora, B., Keinonen, S., Chen, Y., Brinkley, C., Stone, M., Tobler, L., Keating, S., Schito, M., et al. (2014) Development of a contemporary globally diverse HIV viral panel by the EQAPOL program. J. Immunol. Methods 409, 117-130.

(36) Venner, C. M., Nankya, I., Kyeyune, F., Demers, K., Kwok, C., Chen, P.-L., Rwambuya, S., Munjoma, M., Chipato, T., Byamugisha, J., et al. (2016) Infecting HIV-1 Subtype Predicts Disease Progression in Women of Sub-Saharan Africa. EBioMedicine 13, 305-314. 
(37) Hemelaar, J. (2012) The origin and diversity of the HIV-1 pandemic. Trends Mol. Med. 18 (3), 182-192.

(38) Knuchel, M. C., Jullu, B., Shah, C., Tomasik, Z., Stoeckle, M. P., Speck, R. F., Nadal, D., Mshinda, H., Böni, J., Tanner, M., et al. (2007) Adaptation of the ultrasensitive HIV-1 p24 antigen assay to dried blood spot testing. JAIDS, J. Acquired Immune Defic. Syndr. 44 (3), 247-253.

(39) Kabat, E. A., Wu, T. T., Foeller, C., Perry, H. M., and Gottesman, K. S. (1992) Sequences of Proteins of Immunological Interest, DIANE Publishing, Bethesda, MD.

(40) Hinz, A., Lutje Hulsik, D., Forsman, A., Koh, W. W.-L., Belrhali, H., Gorlani, A., De Haard, H., Weiss, R. A., Verrips, T., and Weissenhorn, W. (2010) Crystal structure of the neutralizing Llama $\mathrm{V}(\mathrm{HH}) \mathrm{D} 7$ and its mode of HIV-1 gp120 interaction. PLoS One 5 (5), e10482.

(41) Muyldermans, S., Atarhouch, T., Saldanha, J., Barbosa, J. A., and Hamers, R. (1994) Sequence and structure of VH domain from naturally occurring camel heavy chain immunoglobulins lacking light chains. Protein Eng., Des. Sel. 7 (9), 1129-1135.

(42) Davies, J., and Riechmann, L. (1996) Single antibody domains as small recognition units: design and in vitro antigen selection of camelized, human $\mathrm{VH}$ domains with improved protein stability. Protein Eng., Des. Sel. 9 (6), 531-537.

(43) Briggs, J. A. G., and Kräusslich, H.-G. (2011) The molecular architecture of HIV. J. Mol. Biol. 410 (4), 491-500.

(44) Jacques, D. A., McEwan, W. A., Hilditch, L., Price, A. J., Towers, G. J., and James, L. C. (2016) HIV-1 uses dynamic capsid pores to import nucleotides and fuel encapsidated DNA synthesis. Nature 536 (7616), 349-353.

(45) Wagner, J. M., Zadrozny, K. K., Chrustowicz, J., Purdy, M. D., Yeager, M., Ganser-Pornillos, B. K., and Pornillos, O. (2016) Crystal structure of an HIV assembly and maturation switch. eLife 5, 2072.

(46) Deshmukh, L., Schwieters, C. D., Grishaev, A., Ghirlando, R., Baber, J. L., and Clore, G. M. (2013) Structure and dynamics of fulllength HIV-1 capsid protein in solution. J. Am. Chem. Soc. 135 (43), 16133-16147.

(47) Briggs, J. A. G., Wilk, T., Welker, R., Kräusslich, H.-G., and Fuller, S. D. (2003) Structural organization of authentic, mature HIV-1 virions and cores. EMBO journal 22 (7), 1707-1715.

(48) Bairoch, A., Boeckmann, B., Ferro, S., and Gasteiger, E. (2004) Swiss-Prot: juggling between evolution and stability. Briefings Bioinf. 5 (1), 39-55.

(49) Harmsen, M. M., Ruuls, R. C., Nijman, I. J., Niewold, T. A., Frenken, L. G., and de Geus, B. (2000) Llama heavy-chain V regions consist of at least four distinct subfamilies revealing novel sequence features. Mol. Immunol. 37 (10), 579-590.

(50) Tanha, J., Dubuc, G., Hirama, T., Narang, S. A., and MacKenzie, C. R. (2002) Selection by phage display of llama conventional V(H) fragments with heavy chain antibody $\mathrm{V}(\mathrm{H}) \mathrm{H}$ properties. J. Immunol. Methods 263 (1-2), 97-109.

(51) Pettersen, E. F., Goddard, T. D., Huang, C. C., Couch, G. S., Greenblatt, D. M., Meng, E. C., and Ferrin, T. E. (2004) UCSF Chimera-a visualization system for exploratory research and analysis. J. Comput. Chem. 25 (13), 1605-1612.

(52) Baker, N. A. (2004) Poisson-Boltzmann methods for biomolecular electrostatics. Methods Enzymol. 383, 94-118.

(53) Igonet, S., Vaney, M.-C., Bartonova, V., Helma, J., Rothbauer, U., Leonhardt, H., Stura, E., Krausslich, H. G., and Rey, F. A. Targeting HIV-1 Virion Formation with Nanobodies-Implications for the Design of Assembly Inhibitors; http://www.rcsb.org/pdb/explore. do? structureId $=2 \times \mathrm{xv} 6$.

(54) Hultberg, A., Temperton, N. J., Rosseels, V., Koenders, M., Gonzalez-Pajuelo, M., Schepens, B., Ibañez, L. I., Vanlandschoot, P., Schillemans, J., Saunders, M., et al. (2011) Llama-derived single domain antibodies to build multivalent, superpotent and broadened neutralizing anti-viral molecules. PLoS One 6 (4), e17665.

(55) Strokappe, N., Szynol, A., Aasa-Chapman, M., Gorlani, A., Forsman Quigley, A., Hulsik, D. L., Chen, L., Weiss, R., De Haard, H., and Verrips, T. (2012) Llama antibody fragments recognizing various epitopes of the CD4bs neutralize a broad range of HIV-1 subtypes A, $\mathrm{B}$ and C. PLoS One 7 (3), e33298.

(56) Yoo, S., Myszka, D. G., Yeh, C., McMurray, M., Hill, C. P., and Sundquist, W. I. (1997) Molecular recognition in the HIV-1 capsid/ cyclophilin A complex. J. Mol. Biol. 269 (5), 780-795.

(57) Kabsch, W. (2010) XDS. Acta Crystallogr., Sect. D: Biol. Crystallogr. 66 (2), 125-132.

(58) McCoy, A. J., Grosse-Kunstleve, R. W., Adams, P. D., Winn, M. D., Storoni, L. C., and Read, R. J. (2007) Phaser crystallographic software. J. Appl. Crystallogr. 40 (4), 658-674.

(59) Emsley, P., Lohkamp, B., Scott, W. G., and Cowtan, K. (2010) Features and development of Coot. Acta Crystallogr., Sect. D: Biol. Crystallogr. 66 (4), 486-501.

(60) Murshudov, G. N., Vagin, A. A., and Dodson, E. J. (1997) Refinement of macromolecular structures by the maximum-likelihood method. Acta Crystallogr., Sect. D: Biol. Crystallogr. 53 (3), 240-255.

(61) Adams, P. D., Afonine, P. V., Bunkóczi, G., Chen, V. B., Davis, I. W., Echols, N., Headd, J. J., Hung, L.-W., Kapral, G. J., GrosseKunstleve, R. W., et al. (2010) PHENIX: a comprehensive Pythonbased system for macromolecular structure solution. Acta Crystallogr., Sect. D: Biol. Crystallogr. 66 (2), 213-221.

(62) Phillips, J. C., Braun, R., Wang, W., Gumbart, J., Tajkhorshid, E., Villa, E., Chipot, C., Skeel, R. D., Kalé, L., and Schulten, K. (2005) Scalable molecular dynamics with NAMD. J. Comput. Chem. 26 (16), $1781-1802$

(63) Pronk, S., Páll, S., Schulz, R, Larsson, P., Bjelkmar, P., Apostolov, R., Shirts, M. R., Smith, J. C., Kasson, P. M., van der Spoel, D., et al. (2013) GROMACS 4.5: a high-throughput and highly parallel open source molecular simulation toolkit. Bioinformatics 29 (7), 845854.

(64) Humphrey, W., Dalke, A., and Schulten, K. (1996) VMD: visual molecular dynamics. J. Mol. Graphics 14 (1), 33-38.

(65) Kumari, R., Kumar, R., and Lynn, A. (2014) G-mmpbsa -A GROMACS tool for high-throughput MM-PBSA calculations. J. Chem. Inf. Model. 54 (7), 1951-1962.

(66) Benton, D. J., Martin, S. R., Wharton, S. A., and McCauley, J. W. (2015) Biophysical measurement of the balance of influenza a hemagglutinin and neuraminidase activities. J. Biol. Chem. 290 (10), $6516-6521$

(67) Xiong, X., Coombs, P. J., Martin, S. R., Liu, J., Xiao, H., McCauley, J. W., Locher, K., Walker, P. A., Collins, P. J., Kawaoka, Y., et al. (2013) Receptor binding by a ferret-transmissible H5 avian influenza virus. Nature 497 (7449), 392-396. 